\title{
Centralizers in the Full Transformation Semigroup
}

\author{
João Araújo \\ Universidade Aberta, R. Escola Politécnica, 147 \\ 1269-001 Lisboa, Portugal \\ $\&$ \\ Centro de Álgebra, Universidade de Lisboa \\ 1649-003 Lisboa, Portugal, mjoao@lmc.fc.ul.pt \\ Janusz Konieczny \\ Department of Mathematics, University of Mary Washington \\ Fredericksburg, VA 22401, USA, jkoniecz@umw.edu \\ Communicated by Mikhail Volkov
}

\begin{abstract}
For an arbitrary set $X$ (finite or infinite), denote by $T(X)$ the semigroup of full transformations on $X$. For $\alpha \in T(X)$, let $C(\alpha)=\{\beta \in T(X): \alpha \beta=\beta \alpha\}$ be the centralizer of $\alpha$ in $T(X)$. The aim of this paper is to characterize the elements of $C(\alpha)$. The characterization is obtained by decomposing $\alpha$ as a join of connected partial transformations on $X$ and analyzing the homomorphisms of the directed graphs representing the connected transformations. The paper closes with a number of open problems and suggestions of future investigations.
\end{abstract}

2010 Mathematics Subject Classification: 20M20, 05C20

Keywords and phrases: Full transformations; centralizers; digraph homomorphisms

\section{Introduction}

For a semigroup $S$ and an element $a \in S$, the centralizer $C(a)$ of $a$ in $S$ is defined by $C(a)=\{x \in$ $S: a x=x a\}$. It is clear that $C(a)$ is a subsemigroup of $S$. Let $X$ be a set. We denote by $P(X)$ the semigroup of partial transformations on $X$, that is, the set of all functions $\alpha: A \rightarrow X$, where $A \subseteq X$, with function composition as multiplication. The semigroup $T(X)$ of full transformations on $X$ is the subsemigroup of $P(X)$ consisting of all elements of $P(X)$ whose domain is $X$. Both $P(X)$ and $T(X)$ have the symmetric group $\operatorname{Sym}(X)$ of permutations of $X$ as their group of units.

A significant amount of research has been devoted to studying centralizers in subsemigroups $S$ of $P(X)$ in the case when $X$ is finite. For example, for various $S$ : the elements of centralizers have been described in [14], [28], [31], [34], [35], and [39]; Green's relations and regularity have been determined in [23], [24], and [25]; and some representation theorems have been obtained in [29], [30], and [37]. See also [1] for the semigroup generated by the idempotents of regular centralizers; and [2] for some centralizers related to maps preserving digraphs.

For an infinite $X$, the centralizers of idempotent transformations in $T(X)$ have been studied in [4], [5], and [38]. The cardinalities of $C(\alpha)$, for certain types of $\alpha \in T(X)$, have been established 
for a countable $X$ in [20], [21], and [22]. The second author has investigated the centralizers of transformations in the semigroup $\Gamma(X)$ of injective elements of $T(X)$ [26], [27].

These investigations have been motivated by the fact that if $S$ is a subsemigroup of $P(X)$ that contains the identity $\operatorname{id}_{X}$, then for any $\alpha \in S$, the centralizer $C(\alpha)$ is a generalization of $S$ in the sense that $S=C\left(\operatorname{id}_{X}\right)$. It is therefore of interest to find out which ideas, approaches, and techniques used to study $S$ can be extended to the centralizers of its elements, and how these centralizers differ as semigroups from $S$.

Another reason to study centralizers is that semigroups are nothing but families of commuting maps. We say that two families of maps in $T(X),(\bar{x}: X \rightarrow X)_{x \in X}$ and $(\underline{x}: X \rightarrow X)_{x \in X}$, are linked if for all $x, y \in X$ we have

$$
(y) \underline{x}=(x) \bar{y} .
$$

Linked families of maps induce naturally a groupoid (or magma) $S=(X, \cdot)$ with multiplication defined by

$$
x y=(y) \underline{x}=(x) \bar{y} .
$$

Now we have the following folklore result.

Theorem 1.1. For a non-empty set $X$, let $(\bar{x}: X \rightarrow X)_{x \in X}$ and $(\underline{x}: X \rightarrow X)_{x \in X}$ be two linked families of maps. Then $(X, \cdot)$, the natural groupoid induced by these families of maps, is a semigroup if and only if

$$
(\forall x, y \in X) \underline{x} \in C(\bar{y}) .
$$

Conversely, every semigroup $S$ induces a pair of linked maps $\left(\bar{s}: S^{1} \rightarrow S^{1}\right)_{s \in S}$ and $\left(\underline{s}: S^{1} \rightarrow\right.$ $\left.S^{1}\right)_{s \in S}$ (the images of $S$ under the left and right regular representations [16, page 7]) such that every $\underline{s}$ commutes with every $\bar{t}(s, t \in S)$.

Centralizers of transformations also attract some attention in various areas of mathematical research, for example, in the study of endomorphisms of unary algebras [19], [36]; in the study of commuting graphs [3], [10], [18]; and in the study of automorphism groups of semigroups [2], [6], and [7].

The first step in studying the centralizers in any transformation semigroup is to characterize their elements. A characterization theorem provides a foundation for all subsequent investigations. Such theorems have been provided for some special transformations, for example, for idempotent transformations $\varepsilon \in T(X)$ [5], [24], and for injective transformations [26]. The purpose of this paper is to provide a description of $C(\alpha)$ for a general $\alpha \in T(X)$, where $X$ is an arbitrary set (finite or infinite). The paper will serve as a reference for future research on centralizers of transformations. The reason is that characterization theorems for transformations of special types can easily be obtained as consequences of either our general theorem or various lemmas (see Section 3) that lead to the theorem.

To obtain a characterization of the elements of $C(\alpha)$ for $\alpha \in T(X)$, we first, in Section 2, express any $\alpha \in P(X)$ as a join of connected elements of $P(X)$, which we will call the connected components of $\alpha$. Then we assume that $\alpha \in T(X)$ and further decompose each connected component of $\alpha$ by expressing it as a join of certain basic injective elements of $P(X)$, which we will call cycles, rays, and chains. It turns out that, for $\alpha \in T(X)$, there are three types of connected components, depending on the types of basic partial transformations that occur in their decomposition. In Section 3, we represent a transformation $\alpha \in T(X)$ as a directed graph $D(\alpha)$. For given connected components $\gamma$ and $\delta$ of $\alpha$, we characterize digraph homomorphisms $\phi$ from $D(\gamma)$ to $D(\delta)$, where $D(\gamma)$ and $D(\delta)$ are the subgraphs of $D(\alpha)$ that represent $\gamma$ and $\delta$, respectively. In Section 4, we 
use the results of Sections 2 and 3 to characterize the elements of $C(\alpha)$ for an arbitrary $\alpha \in T(X)$. Finally, in Section 6, we outline a research program aimed at generalizing, for the centralizers of some particular transformations, many of the structure results proved for $T(X)$.

\section{Decomposition of Full Transformations}

In this section, we introduce the notion of the connected partial transformation on $X$ and prove that every $\alpha \in P(X)$ can be decomposed uniquely as a join of connected $\gamma \in P(X)$ (called connected components of $\alpha$ ). We then introduce the concept of the basic partial transformation and prove that each connected component of $\alpha \in T(X)$ can be further decomposed (although not uniquely) as a join of basic partial transformations. Depending on this decomposition, each connected component of $\alpha$ will be of one of three distinguished types.

Let $\gamma \in P(X)$. We denote the domain of $\gamma$ by $\operatorname{dom}(\gamma)$ and the image of $\gamma$ by $\operatorname{im}(\gamma)$. The union $\operatorname{dom}(\gamma) \cup \operatorname{im}(\gamma)$ will be called the span of $\gamma$ and denoted $\operatorname{span}(\gamma)$.

We will write mappings on the right and compose from left to right; that is, for $f: A \rightarrow B$ and $g: B \rightarrow C$, we will write $x f$, rather than $f(x)$, and $x(f g)$, rather than $g(f(x))$.

Notation 2.1. From now on, we will fix a nonempty set $X$ and an element $\diamond$ and assume that $\diamond \notin X$. For $\gamma \in P(X)$ and $x \in X$, we will write $x \gamma=\diamond$ if and only if $x \notin \operatorname{dom}(\gamma)$. We will also assume that $\diamond \gamma=\diamond$. With this notation, it will make sense to write $x \gamma=y \delta$ or $x \gamma \neq y \delta(\gamma, \delta \in P(X)$, $x, y \in X)$ even when $x \notin \operatorname{dom}(\gamma)$ or $y \notin \operatorname{dom}(\delta)$. We will denote by 0 the partial transformation on $X$ that has empty set as its domain.

Definition 2.2. An element $\gamma \in P(X)$ is called connected if $\gamma \neq 0$ and for all $x, y \in \operatorname{span}(\gamma)$, $x \gamma^{k}=y \gamma^{m} \neq \diamond$ for some integers $k, m \geq 0$ (where $\gamma^{0}=\mathrm{id}_{X}$ ).

Definition 2.3. Let $\gamma, \delta \in P(X)$. We say that $\gamma$ and $\delta$ are compatible if $x \gamma=x \delta$ for all $x \in$ $\operatorname{dom}(\gamma) \cap \operatorname{dom}(\delta)$; they are disjoint if $\operatorname{dom}(\gamma) \cap \operatorname{dom}(\delta)=\emptyset$; and they are completely disjoint if $\operatorname{span}(\gamma) \cap \operatorname{span}(\delta)=\emptyset$.

Definition 2.4. Let $C$ be a set of pairwise compatible elements of $P(X)$. The join of the elements of $C$, denoted $\bigsqcup_{\gamma \in C} \gamma$, is an element of $P(X)$ defined by

$$
x\left(\bigsqcup_{\gamma \in C} \gamma\right)= \begin{cases}x \gamma & \text { if } x \in \operatorname{dom}(\gamma) \text { for some } \gamma \in C, \\ \diamond & \text { otherwise. }\end{cases}
$$

If $C=\left\{\gamma_{1}, \gamma_{2}, \ldots, \gamma_{k}\right\}$ is finite, we may write $\bigsqcup_{\gamma \in C} \gamma$ as $\gamma_{1} \sqcup \gamma_{2} \sqcup \cdots \sqcup \gamma_{k}$.

Let $\gamma \in P(X)$. We will write $x \stackrel{\gamma}{\rightarrow} y$ to mean that $x \in \operatorname{dom}(\gamma)$ and $x \gamma=y$. For $\delta \in P(X)$, we say that $\delta$ is contained in $\gamma$ (or $\gamma$ contains or has $\delta$ ), and write $\delta \sqsubset \gamma$, if $\operatorname{dom}(\delta) \subseteq \operatorname{dom}(\gamma)$ and $x \delta=x \gamma$ for every $x \in \operatorname{dom}(\delta)$.

For a mapping $f: A \rightarrow B$ and $A_{1} \subseteq A$, we denote by $\left.f\right|_{A_{1}}$ the restriction of $f$ to $A_{1}$, and by $A_{1} f$ the image of $A_{1}$ under $f$.

Proposition 2.5. Let $\alpha \in P(X)$ with $\alpha \neq 0$. Then there exists a unique set $C$ of pairwise completely disjoint, connected elements of $P(X)$ such that $\alpha=\bigsqcup_{\gamma \in C} \gamma$. 
Proof. Define a relation $\rho$ on $\operatorname{dom}(\alpha)$ by: $(x, y) \in \rho$ if $x \alpha^{k}=y \alpha^{m} \neq \diamond$ for some integers $k, m \geq 0$. It is clear that $\rho$ is an equivalence relation on $\operatorname{dom}(\alpha)$. Let $J$ be a complete set of representatives of the equivalence classes of $\rho$. For every $x \in J$, let $\gamma_{x}=\left.\alpha\right|_{x \rho}$, where $x \rho$ is the $\rho$-equivalence class of $x$. By the definition of $\rho$, each such $\gamma_{x}$ is connected, and $\gamma_{x}$ and $\gamma_{y}$ are completely disjoint for all $x, y \in J$ with $x \neq y$. Then the set $C=\left\{\gamma_{x}: x \in J\right\}$ consists of pairwise completely disjoint, connected transformations contained in $\alpha$, and $\alpha=\bigsqcup_{\gamma \in C} \gamma$.

Suppose $D$ is any set of pairwise completely disjoint, connected transformations contained in $\alpha$ such that $\alpha=\bigsqcup_{\delta \in D} \delta$. Let $\delta \in D$ and let $y \in \operatorname{dom}(\delta)$. Then $y \in x \rho$ for some $x \in J$. We want to prove that $\delta=\gamma_{x}$. Let $z \in \operatorname{dom}(\delta)$. Since $\delta$ is connected, $y \delta^{k}=z \delta^{m} \neq \diamond$ for some $k, m \geq 0$. But then, since $\delta$ is contained in $\alpha$, we have $y \alpha^{k}=z \alpha^{m} \neq \diamond$. Hence $(y, z) \in \rho$, and so $z \in y \rho=x \rho=\operatorname{dom}\left(\gamma_{x}\right)$. We proved that $\operatorname{dom}(\delta) \subseteq \operatorname{dom}\left(\gamma_{x}\right)$.

Suppose to the contrary that $\operatorname{dom}\left(\gamma_{x}\right)$ is not included in $\operatorname{dom}(\delta)$, that is, that there is $w \in$ $\operatorname{dom}\left(\gamma_{x}\right)$ such that $w \notin \operatorname{dom}(\delta)$. Since $\gamma_{x}$ is connected, $w \gamma_{x}^{p}=x \gamma_{x}^{q} \neq \diamond$ for some $p, q \geq 0$. Let $y_{i}=y \gamma_{x}^{i}=y \alpha^{i}$ and $w_{j}=w \gamma_{x}^{j}=w \alpha^{j}$ for $i=0,1, \ldots, p$ and $j=0,1, \ldots, q$. Then $y_{p}=w_{q}$ and let $u=y_{p}=w_{q}$. With this notation, we have

$$
y=y_{0} \stackrel{\alpha}{\rightarrow} y_{1} \stackrel{\alpha}{\rightarrow} \cdots \stackrel{\alpha}{\rightarrow} y_{p}=u \text { and } w=w_{0} \stackrel{\alpha}{\rightarrow} w_{1} \stackrel{\alpha}{\rightarrow} \cdots \stackrel{\alpha}{\rightarrow} w_{q}=u .
$$

Since $w \in \operatorname{dom}\left(\gamma_{x}\right) \subseteq \operatorname{dom}(\alpha)$, there is $\delta_{1} \in D$ such that $w \in \operatorname{dom}\left(\delta_{1}\right)$. We claim that $\left\{y_{0}, y_{1}, \ldots, y_{p-1}\right\} \subseteq \operatorname{dom}(\delta)$. If not, then, since $y_{0}=y \in \operatorname{dom}(\delta)$, there would be some $i \in\{0,1, \ldots, p-2\}$ such that $y_{i} \in \operatorname{dom}(\delta)$ and $y_{i+1} \notin \operatorname{dom}(\delta)$. But $y_{i+1} \in \operatorname{dom}(\alpha)$, and so $y_{i+1} \in \operatorname{dom}\left(\delta_{2}\right)$ for some $\delta_{2} \in D$. We would then have $\delta \neq \delta_{2}$ and $y_{i+1} \in \operatorname{span}(\delta) \cap \operatorname{span}\left(\delta_{2}\right)$, which is impossible since $\delta$ and $\delta_{2}$ are completely disjoint. The claim has been proved. By the same argument applied to $\delta_{1}$ and $\left\{w_{0}, w_{1}, \ldots, w_{q-1}\right\}$, we obtain $\left\{w_{0}, w_{1}, \ldots, w_{q-1}\right\} \subseteq \operatorname{dom}\left(\delta_{1}\right)$. Thus

$$
y_{p-1} \delta=y_{p-1} \alpha=y_{p}=u=w_{q}=w_{q-1} \alpha=w_{q-1} \delta_{1} .
$$

Thus we have $\delta \neq \delta_{1}$ with $u \in \operatorname{im}(\delta) \cap \operatorname{im}\left(\delta_{1}\right)$, which is a contradiction since $\delta$ and $\delta_{1}$ are completely disjoint. We proved that $\operatorname{dom}\left(\gamma_{x}\right) \subseteq \operatorname{dom}(\delta)$, and so $\operatorname{dom}(\delta)=\operatorname{dom}\left(\gamma_{x}\right)$. Now for all $v \in \operatorname{dom}(\delta)=\operatorname{dom}\left(\gamma_{x}\right)$, we have $v \delta=v \alpha=v \gamma_{x}$, and so $\delta=\gamma_{x} \in C$. We proved that $D \subseteq C$.

For the reverse inclusion, let $\gamma_{x}$ be an arbitrary element of $C$. Select $y \in \operatorname{dom}\left(\gamma_{x}\right)$. Then, there is $\delta \in D$ such that $y \in \operatorname{dom}(\delta)$. By the foregoing argument, we have $\delta=\gamma_{x}$, and so $\gamma_{x} \in D$. Hence $C \subseteq D$, and so $D=C$. We proved that the set $C$ is unique, which completes the proof.

Let $\alpha \in T(X)$. The elements of the set $C$ from Proposition 2.5 will be called the connected components of $\alpha$. This use of graph theory terminology is intentional since $\alpha$ can be represented by the directed graph $D(\alpha)=(X, \alpha)$, where $(x, y)$ is an arc in $D(\alpha)$ if and only if $x \alpha=y$. (See Section 3 for details.) Then the connected components of $\alpha$ correspond to the connected components of the underlying undirected graph of $D(\alpha)$.

Regarding directed graphs, we will adopt the convention that the arrows will be deleted with the understanding that the arrow goes up along the edge, to the right if the edge is horizontal, and the arrows go counter-clockwise along a cycle. For example, the digraph in Figure 2.1 represents the transformation

$$
\alpha=\left(\begin{array}{cccccccccc}
1 & 2 & 3 & 4 & 5 & 6 & 7 & 8 & 9 & \ldots \\
2 & 3 & 1 & 1 & 1 & 5 & 8 & 9 & 10 & \ldots
\end{array}\right) \in T(X),
$$

where $X=\{1,2,3, \ldots\}$.

The connected components of $\alpha \in T(X)$ further decompose into basic elements in $P(X)$. 


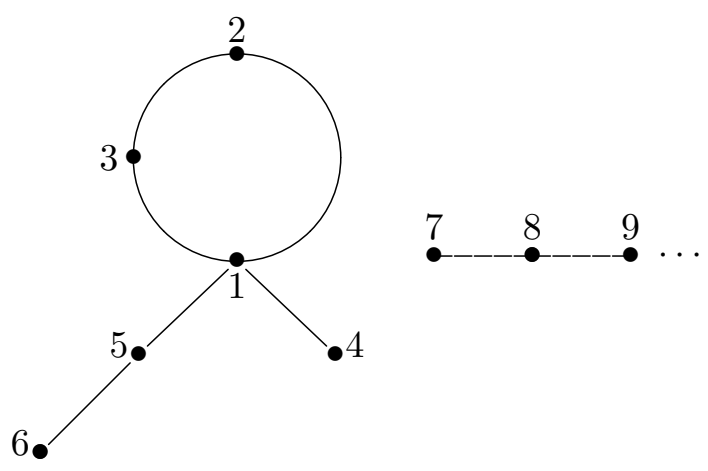

Figure 2.1: The digraph of a transformation.

Definition 2.6. Let $\ldots, x_{-2}, x_{-1}, x_{0}, x_{1}, x_{2}, \ldots$ be pairwise distinct elements of $X$. The following elements of $P(X)$ will be called basic partial transformations on $X$ (see Figure 2.2).

- A cycle of length $k(k \geq 1)$, written $\left(x_{0} x_{1} \ldots x_{k-1}\right)$, is an element $\sigma \in P(X)$ with $\operatorname{dom}(\sigma)=\left\{x_{0}, x_{1}, \ldots, x_{k-1}\right\}, x_{i} \sigma=x_{i+1}$ for all $0 \leq i<k-1$, and $x_{k-1} \sigma=x_{0}$.

- A right ray, written $\left[x_{0} x_{1} x_{2} \ldots\right\rangle$, is an element $\eta \in P(X)$ with $\operatorname{dom}(\eta)=\left\{x_{0}, x_{1}, x_{2}, \ldots\right\}$ and $x_{i} \eta=x_{i+1}$ for all $i \geq 0$.

- A double ray, written $\left\langle\ldots x_{-2} x_{-1} x_{0} x_{1} x_{2} \ldots\right\rangle$, is an element $\omega \in P(X)$ with $\operatorname{dom}(\omega)=$ $\left\{\ldots, x_{-2}, x_{-1}, x_{0}, x_{1}, x_{2}, \ldots\right\}$ and $x_{i} \omega=x_{i+1}$ for all $i$.

- A left ray, written $\left\langle\ldots x_{2} x_{1} x_{0}\right]$, is an element $\lambda \in P(X)$ with $\operatorname{dom}(\lambda)=\left\{x_{1}, x_{2}, x_{3}, \ldots\right\}$ and $x_{i} \lambda=x_{i-1}$ for all $i>0$.

- A chain of length $k(k \geq 1)$, written $\left[x_{0} x_{1} \ldots x_{k}\right]$, is an element $\tau \in P(X)$ with $\operatorname{dom}(\tau)=$ $\left\{x_{0}, x_{1}, \ldots, x_{k-1}\right\}$ and $x_{i} \tau=x_{i+1}$ for all $0 \leq i \leq k-1$.

By a ray we will mean a double, right, or left ray.

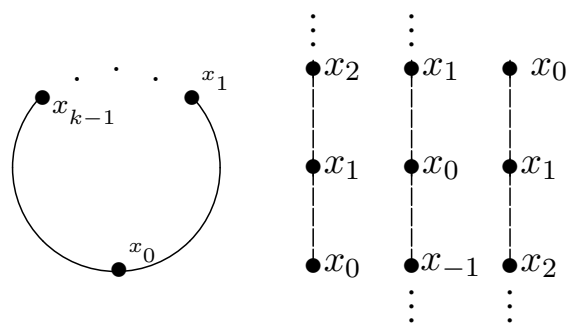

Figure 2.2: Basic partial transformations.

We note the following:

(i) All basic partial transformations are connected and injective.

(ii) The span of a basic partial transformation is exhibited by the notation. For example, the span of the right ray $[123 \ldots\rangle$ is $\{1,2,3, \ldots\}$. 
(iii) The left bracket in " $\varepsilon=[x \ldots$." indicates that $x \notin \operatorname{im}(\varepsilon)$; while the right bracket in " $\varepsilon=$ $\ldots x]$ " indicates that $x \notin \operatorname{dom}(\varepsilon)$. For example, for the chain $\tau=[1234], \operatorname{dom}(\tau)=$ $\{1,2,3\}$ and $\operatorname{im}(\tau)=\{2,3,4\}$.

(iv) A cycle $\left(x_{0} x_{1} \ldots x_{k-1}\right)$ differs from the corresponding cycle in the symmetric group of permutations on $X$ in that the former is undefined for every $x \in X-\left\{x_{0}, x_{1}, \ldots, x_{k-1}\right\}$ while the latter is fixed for every such $x$.

We will now analyze which combinations of basic transformations can occur in a connected component of $\alpha \in T(X)$.

Definition 2.7. A right ray $\eta=\left[x_{0} x_{1} x_{2} \ldots\right\rangle$ contained in $\alpha \in T(X)$ is called a maximal right ray in $\alpha$ if $x_{0} \notin \operatorname{im}(\alpha)$.

For example, consider $\alpha=[4567 \ldots\rangle \sqcup[1236] \in T(\mathbb{N})$, where $\mathbb{N}$ is the set of positive integers. Then $\alpha$ contains infinitely many right rays, for example $[3678 \ldots\rangle$ and $[78910 \ldots\rangle$, but only two of them, namely $[4567 \ldots\rangle$ and $[123678 \ldots\rangle$ are maximal. Note also that $\alpha$ is connected.

Lemma 2.8. Let $\gamma$ be a connected component of $\alpha \in T(X)$. Then:

(1) If $\gamma$ has a cycle $\left(x_{0} x_{1} \ldots x_{k-1}\right)$, then for every $x \in \operatorname{dom}(\gamma), x \gamma^{m}=x_{0}$ for some $m \geq 0$.

(2) If $\gamma$ has a right ray $\left[x_{0} x_{1} x_{2} \ldots\right\rangle$ or a double ray $\left\langle\ldots x_{-1} x_{0} x_{1} \ldots\right\rangle$, then for every $x \in$ $\operatorname{dom}(\gamma), x \gamma^{m}=x_{i}$ for some $m, i \geq 0$.

Proof. Suppose $\gamma$ has a cycle $\left(x_{0} x_{1} \ldots x_{k-1}\right)$ and let $x \in \operatorname{dom}(\gamma)$. Since $\gamma$ is connected, $x \gamma^{p}=$ $x_{0} \gamma^{q}$ for some $p, q \geq 0$. Since $x_{0}$ lies on the cycle $\left(x_{0} x_{1} \ldots x_{k-1}\right)$, we may assume that $0 \leq q \leq$ $k-1$. Thus for $m=p+k-q$, we have

$$
x \gamma^{m}=x \gamma^{p+k-q}=\left(x \gamma^{p}\right) \gamma^{k-q}=\left(x_{0} \gamma^{q}\right) \gamma^{k-q}=x^{q} \gamma^{k-q}=x_{0} .
$$

Suppose $\gamma$ has a right ray $\left[x_{0} x_{1} x_{2} \ldots\right\rangle$ and let $x \in \operatorname{dom}(\gamma)$. Since $\gamma$ is connected, $x \gamma^{m}=$ $x_{0} \gamma^{i}=x_{i}$ for some $m, i \geq 0$. A proof in the case of a double ray is the same.

Lemma 2.9. Let $\gamma$ be a connected component of $\alpha \in T(X)$ and let $x \in \operatorname{im}(\gamma)$ such that $x$ does not lie on a cycle in $\gamma$. Then $\gamma$ contains a left ray $\left\langle\ldots y_{3} y_{2} y_{1} x\right]$ or a chain $\left[y_{k} y_{k-1} \ldots y_{1} x\right](k \geq 1)$ with $y_{k} \notin \operatorname{im}(\gamma)$.

Proof. Since $x \in \operatorname{im}(\gamma)$, there is $y_{1} \in X$ such that $y_{1} \gamma=x_{0}$. If $y_{1} \in \operatorname{im}(\gamma)$, then $y_{2} \gamma=y_{1}$ for some $y_{2} \in X$. Continuing this way, we either arrive at $y_{k} \in X$ such that $y_{k} \gamma=y_{k-1}$ and $y_{k} \notin \operatorname{im}(\gamma)$ or the process of constructing $y_{1}, y_{2}, y_{3}, \ldots$ will go on forever. Note that there will be no repetition in the sequence $\left\langle y_{i}\right\rangle$ (finite or infinite) since $x$ does not lie on a cycle in $\gamma$. Hence, either $\gamma$ contains a left ray $\left\langle\ldots y_{3} y_{2} y_{1} x\right]$ or a desired chain.

Proposition 2.10. Let $\gamma$ be a connected component of $\alpha \in T(X)$. Then:

(1) If $\gamma$ has a cycle, then the cycle is unique and $\gamma$ does not have any double or right rays.

(2) If $\gamma$ does not have a cycle, then $\gamma$ is a join of the double rays and maximal right rays contained in $\gamma$. 
Proof. Suppose that $\gamma$ has a cycle, say $\sigma=\left(x_{0} x_{1} \ldots x_{k-1}\right)$. Let $\theta=\left(y_{0} y_{1} \ldots y_{m-1}\right)$ be any cycle in $\gamma$. We want to prove that $\sigma=\theta$. We may assume that $k \leq m$. By Lemma 2.8, $y_{0} \gamma^{p}=x_{0}$ for some $p \geq 0$. On the other hand, $y_{0} \gamma^{p}=y_{j}$ for some $j \in\{0, \ldots, m-1\}$, and so $x_{0}=y_{j}$. Since we can rewrite $\theta$ as $\left(y_{j} y_{j+1} \ldots y_{j-1}\right)$, we may assume that $y_{j}=y_{0}$, so $x_{0}=y_{0}$. But then $x_{i}=x_{0} \gamma^{i}=y_{0} \gamma^{i}=y_{i}$ for every $i \in\{0, \ldots, k-1\}$ and $y_{k-1} \gamma=x_{k-1} \gamma=x_{0}=y_{0}$. It follows that $k=m$ and $\sigma=\theta$. We proved that a cycle in $\gamma$ is unique.

Suppose that $\gamma$ with a cycle $\left(x_{0} x_{1} \ldots x_{k-1}\right)$ also has a double ray, say $\left\langle\ldots y_{-1} y_{0} y_{1} \ldots\right\rangle$. By Lemma 2.8, $y_{0} \gamma^{m}=x_{0}$ for some $m \geq 0$. But then $y_{0} \gamma^{m+k}=\left(y_{0} \gamma^{m}\right) \gamma^{k}=x_{0} \gamma^{k}=x_{0}=y_{0}$, which is a contradiction since $y_{0} \gamma^{m+k}=y_{m+k} \neq y_{0}$ (since $m \geq 0$ and $k \geq 1$ ). Thus $\gamma$ does not have a double ray. Similarly, $\gamma$ cannot have a right ray. We have proved (1).

To prove (2), suppose $\gamma$ does not have a cycle. Let $R$ be the set of all double and maximal right rays contained in $\gamma$. Then clearly $\bigsqcup_{\varepsilon \in R} \varepsilon \sqsubset \gamma$. (Note that if $\delta_{1}, \delta_{2}$ are contained in $\gamma$, then $\delta_{1}$ and $\delta_{2}$ are compatible since for every $x \in \operatorname{dom}\left(\delta_{1}\right) \cap \operatorname{dom}\left(\delta_{2}\right), x \delta_{1}=x \gamma=x \delta_{2}$.) Select any $x \in \operatorname{dom}(\gamma)$. Then $x_{0}=x, x_{1}=x \gamma, x_{2}=x \gamma^{2}, \ldots$ are pairwise distinct since otherwise $\gamma$ would have a cycle. Consider the right ray $\eta=\left[x_{0} x_{1} x_{2} \ldots\right\rangle$ in $\gamma$. If $\eta$ is not maximal (that is, $x_{0} \in \operatorname{im}(\gamma)$ ), then $\gamma$ contains a left ray $\left\langle\ldots y_{3} y_{2} y_{1} x_{0}\right]$ or a chain $\left[y_{k} y_{k-1} \ldots y_{1} x_{0}\right](k \geq 1)$ with $y_{k} \notin \operatorname{im}(\gamma)$ (by Lemma 2.9). In the former case, $\gamma$ has a double ray $\left\langle\ldots y_{2} y_{1} x_{0} x_{1} x_{2} \ldots\right\rangle$; and in the latter case $\gamma$ has a maximal right ray $\left[y_{k} \ldots y_{1} x_{0} x_{1} x_{2} \ldots\right\rangle$. Thus $x=x_{0} \in \operatorname{dom}(\varepsilon)$ for some $\varepsilon \in R$, and it follows that $\gamma=\bigsqcup_{\varepsilon \in R} \varepsilon$.

Definition 2.11. Let $\gamma$ be a connected component of $\alpha \in T(X)$. By Proposition 2.10, exactly one of the following three conditions holds (see Figures 2.3, 2.4, and 2.5):

(i) $\gamma$ contains a unique cycle;

(ii) $\gamma$ contains a double ray;

(iii) $\gamma$ does not contain a double ray and $\gamma$ is the join of its maximal right rays.

If $\gamma$ satisfies (iii), we will say that $\gamma$ is of type $\operatorname{rro}$ ("right rays only").

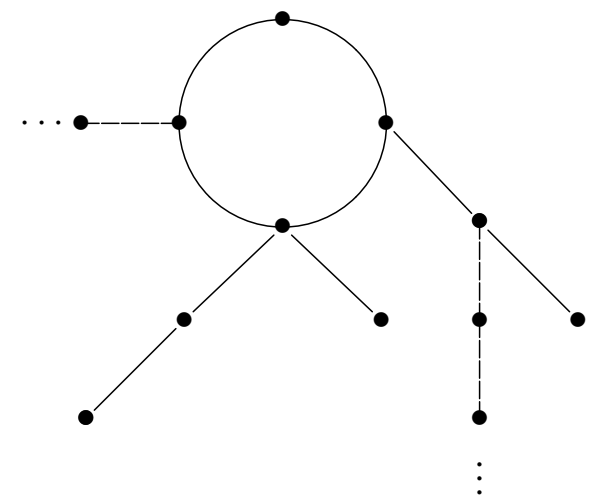

Figure 2.3: A connected component with a cycle.

Let $\varepsilon \in P(X)$ be a basic partial transformation. If $x \in \operatorname{span}(\varepsilon)$, we will say that $x$ lies on $\varepsilon$.

Definition 2.12. Let $\gamma$ be a connected component of $\alpha \in T(X)$. 


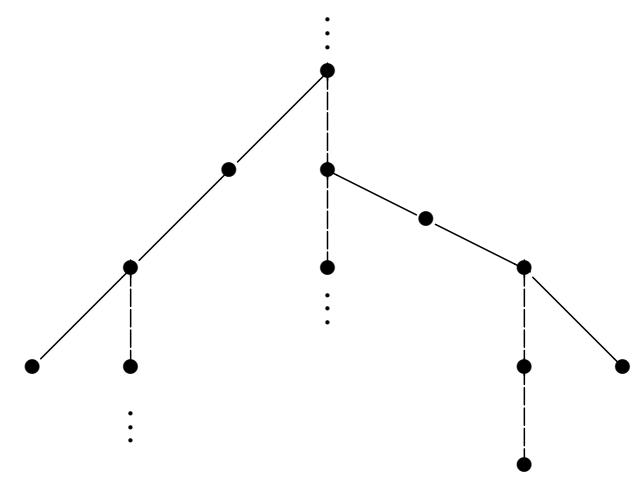

Figure 2.4: A connected component with a double ray.

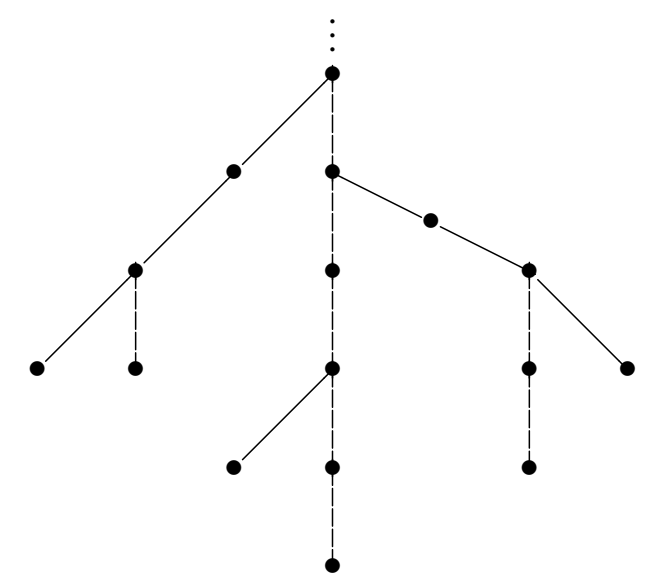

Figure 2.5: A connected component of type rro.

- A chain $\left[y_{0} y_{1} \ldots y_{m}\right]$ in $\gamma$ is called a finite branch of a cycle $\sigma$ [double ray $\omega$, right ray $\eta$ ] in $\gamma$ if $y_{0} \notin \operatorname{im}(\gamma), y_{m}$ lies on $\sigma[\omega, \eta]$, and $y_{m-1}$ does not lie on $\sigma[\omega, \eta]$.

- A left ray $\left\langle\ldots y_{2} y_{1} y_{0}\right.$ ] in $\gamma$ is called an infinite branch of a cycle $\sigma$ [double ray $\omega$ ] in $\gamma$ if $y_{0}$ lies on $\sigma[\omega]$ and $y_{1}$ does not lie on $\sigma[\omega]$.

By a branch we will mean a finite or infinite branch. We will use the notation $\left(\ldots y_{2} y_{1} y_{0}\right]$ for a branch that is finite or infinite (but we do not know which).

If $\sigma=\left(x_{0} x_{1} \ldots x_{k-1}\right)$ is a cycle in $\gamma$ and $\varepsilon$ is a branch of $\sigma$ with the terminal point $x_{i}$, we will say that $\varepsilon$ is a branch of $\sigma$ at $x_{i}$. (If $\varepsilon$ is a left ray or a chain, then the terminal point of $\varepsilon$ is the element $x \in X$ such that $x \in \operatorname{im}(\varepsilon)-\operatorname{dom}(\varepsilon)$.) We will use a similar language for branches of a double ray $\omega$ and a right ray $\eta$. Note that all branches of a right ray $\eta$ in $\gamma$ are finite by definition.

For example, the cycle in Figure 2.3 has two infinite branches (at the same point) and four finite branches (at three different points). The transformation whose digraph is presented in Figure 2.4 has two double rays. The vertical double ray has one infinite branch and three finite branches (at two different points). The transformation from Figure 2.5 is connected of type rro with seven maximal right rays. The vertical maximal right ray has six (finite) branches (at three different points). 
Proposition 2.13. Let $\gamma$ be a connected component of $\alpha \in T(X)$. Then:

(1) If $\gamma$ has a (unique) cycle $\sigma$, then $\gamma$ is the join of $\sigma$ and its branches.

(2) If $\gamma$ has a double ray $\omega$, then $\gamma$ is the join of $\omega$ and its branches.

(3) If $\gamma$ is of type rro with a maximal right ray $\eta$, then $\gamma$ is the join of $\eta$ and its (finite) branches.

Proof. Suppose $\gamma$ has a cycle $\sigma$. Let $x \in \operatorname{dom}(\gamma)$ be such that $x$ does not lie on $\sigma$. Let $t$ be the smallest positive integer such that $x \gamma^{t}$ lies on $\sigma$ (such a $t$ exists by Lemma 2.8). If $x \notin \operatorname{im}(\gamma)$, then $\left[x x \gamma \ldots x \gamma^{t}\right]$ is a finite branch of $\sigma$. If $x \in \operatorname{im}(\gamma)$, then $\gamma$ contains a left ray $\left\langle\ldots y_{3} y_{2} y_{1} x\right]$ or a chain $\left[y_{k} y_{k-1} \ldots y_{1} x\right](k \geq 1)$ with $y_{k} \notin \operatorname{im}(\gamma)$ (by Lemma 2.9). In the former case, $\left\langle\ldots y_{2} y_{1} x x \gamma \ldots x \gamma^{t}\right]$ is an infinite branch of $\sigma$; and in the latter case $\left[y_{k} \ldots y_{1} x x \gamma \ldots x \gamma^{t}\right]$ is a finite branch of $\sigma$. Thus every element of $\operatorname{dom}(\gamma)$ either lies on $\sigma$ or on one of the branches of $\sigma$, which proves (1). The proofs of (2) and (3) are similar. We note that the foregoing argument applied to $\eta$ in (3) will not produce and infinite branch since $\gamma$ of type rro does not contain a double ray.

\section{Digraph Homomorphisms}

In this section, we represent $\alpha \in T(X)$ and its connected components as directed graphs. For given connected components $\gamma$ and $\delta$ of $\alpha$, we investigate digraph homomorphisms from $D(\gamma)$ to $D(\delta)$. This approach is justified by Proposition 3.1 below.

A directed graph (or a digraph) is a pair $D=(X, \rho)$ where $X$ is a non-empty set (not necessarily finite) and $\rho$ is a binary relation on $X$. Any element $x \in X$ is called a vertex of $D$, and any pair $(x, y) \in \rho$ is called an arc of $D$. Let $D_{1}=\left(X_{1}, \rho_{1}\right)$ and $D_{2}=\left(X_{2}, \rho_{2}\right)$ be digraphs. A mapping $\phi: X_{1} \rightarrow X_{2}$ is called a homomorphism from $D_{1}$ to $D_{2}$ if it preserves edges, that is, for all $x, y \in X_{1}$, if $(x, y) \in \rho_{1}$, then $(x \phi, y \phi) \in \rho_{2}$ [13]. We say that $D_{1}$ is homomorphic to $D_{2}$ if there is a homomorphism from $D_{1}$ to $D_{2}$.

Let $\alpha \in T(X)$. Then $\alpha$ can be represented by the directed graph $D(\alpha)=(X, \alpha)$, where $\alpha$ is viewed as a binary relation on $X$. In other words, for all $x, y \in X,(x, y)$ is an arc in $D(\alpha)$ if and only if $x \alpha=y$. Let $\gamma$ be a connected component of $\alpha$. By $D(\gamma)$ we will mean the directed subgraph of $D(\alpha)$ induced by $\operatorname{dom}(\gamma)$. That is, $\operatorname{dom}(\gamma)$ is the set of vertices of $D(\gamma)$ and for all $x, y \in \operatorname{dom}(\gamma),(x, y)$ is an arc in $D(\gamma)$ if and only if $(x, y)$ is an arc in $D(\alpha)$. (The latter is equivalent to $x \gamma=y$.) If $(x, y)$ is an arc in $D(\gamma)$, we will write $x \stackrel{\gamma}{\rightarrow} y$ (or $x \rightarrow y$ if no ambiguity arises). The same convention will apply to the digraph $D(\alpha)$.

The following proposition provides a link between centralizers of elements of $T(X)$ and digraph homomorphisms.

Proposition 3.1. Let $\alpha, \beta \in T(X)$. Then $\beta \in C(\alpha)$ if and only if $\beta$ is a homomorphism from $D(\alpha)$ to $D(\alpha)$.

Proof. Suppose $\beta \in C(\alpha)$. Let $x \rightarrow y$ be an arc in $D(\alpha)$, that is, $y=x \alpha$. Then, since $\alpha \beta=\beta \alpha$, we have $(x \beta) \alpha=x(\beta \alpha)=x(\alpha \beta)=(x \alpha) \beta=y \beta$, and so $x \beta \rightarrow y \beta$. Hence $\beta$ is a homomorphism from $D(\alpha)$ to $D(\alpha)$.

Conversely, suppose that $\beta$ is a homomorphism from $D(\alpha)$ to $D(\alpha)$. Let $x \in X$. Then $x \beta \rightarrow$ $(x \alpha) \beta$ since $x \rightarrow x \alpha$ and $\beta$ preserves edges. But $x \beta \rightarrow(x \alpha) \beta$ means that $(x \beta) \alpha=(x \alpha) \beta$, which implies $x(\alpha \beta)=x(\beta \alpha)$. Hence $\alpha \beta=\beta \alpha$, and so $\beta \in C(\alpha)$. 
For the remainder of this section, we will work on characterizing digraph homomorphisms $\phi$ from $D(\gamma)$ to $D(\delta)$, where $\gamma$ and $\delta$ are connected components of $\alpha \in T(X)$. It will be convenient to introduce the following definitions. We agree that if $\theta=\left(y_{0} \ldots y_{m-1}\right)$ is a cycle and $i$ is an integer, then $y_{i}$ means $y_{r}$ where $r \equiv i(\bmod m)$ and $0 \leq r<m$.

Definition 3.2. Let $\phi \in P(X)$.

- Let $\sigma=\left(x_{0} \ldots x_{k-1}\right)$ and $\theta=\left(y_{0} \ldots y_{m-1}\right)$ be cycles such $\operatorname{dom}(\sigma) \subseteq \operatorname{dom}(\phi)$. We say that $\phi$ wraps $\sigma$ around $\theta$ at $y_{t}$ if $x_{i} \phi=y_{t+i}$ for all $0 \leq i \leq k-1$. (See Figure 3.1.)

- Let $\tau=\left[z_{0} \ldots z_{p}\right]$ be a chain and $\theta=\left(y_{0} \ldots y_{m-1}\right)$ be a cycle such that $\operatorname{span}(\tau) \subseteq \operatorname{dom}(\phi)$. We say that $\phi$ wraps $\tau$ around $\theta$ at $y_{t}$ if $z_{i} \phi=y_{t+i}$ for all $0 \leq i \leq p$. (See Figure 3.2.)

- Let $\lambda=\left\langle\ldots z_{2} z_{1} z_{0}\right]$ be a left ray and $\theta=\left(y_{0} \ldots y_{m-1}\right)$ be a cycle such that $\operatorname{span}(\lambda) \subseteq$ $\operatorname{dom}(\phi)$. We say that $\phi$ counter-wraps $\lambda$ around $\theta$ at $y_{t}$ if $z_{i} \phi=y_{t-i}$ for all $i \geq 0$. (See Figure 3.3.)

- Let $\omega=\left\langle\ldots x_{-1} x_{0} x_{1} \ldots\right\rangle$ be a double ray and $\theta=\left(y_{0} \ldots y_{m-1}\right)$ be a cycle such that $\operatorname{dom}(\omega) \subseteq \operatorname{dom}(\phi)$. We say that $\phi$ double-wraps $\omega$ around $\theta$ at $y_{t}$ if $x_{i} \phi=y_{t+i}$ for all $i$. (See Figure 3.4.)

- Let $\eta=\left[x_{0} x_{1} x_{3} \ldots\right\rangle$ be a right ray and $\theta=\left(y_{0} \ldots y_{m-1}\right)$ be a cycle such that $\operatorname{dom}(\eta) \subseteq$ $\operatorname{dom}(\phi)$. We say that $\phi$ wraps $\eta$ around $\theta$ at $y_{t}$ if $x_{i} \phi=y_{t+i}$ for all $i \geq 0$. (See Figure 3.5.)

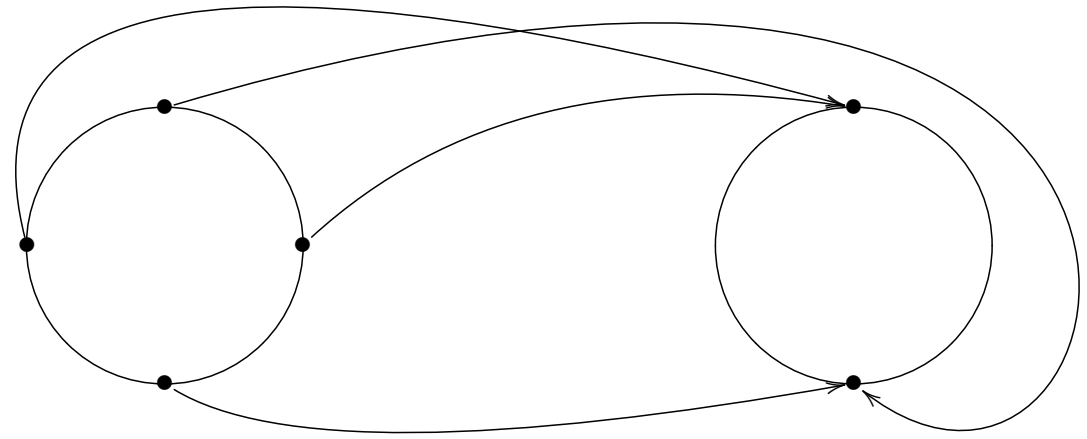

Figure 3.1: A cycle wrapped around a cycle.

Definition 3.3. Let $\phi \in P(X)$.

- Let $\tau=\left[z_{0} \ldots z_{p}\right]$ and $\kappa=\left[w_{0} \ldots w_{p}\right]$ be chains of the same length such that $\operatorname{span}(\tau) \subseteq$ $\operatorname{dom}(\phi)$. We say that $\phi$ maps $\tau$ onto $\kappa$ if $z_{i} \phi=w_{i}$ for all $0 \leq i \leq p$.

- Let $\lambda=\left\langle\ldots z_{2} z_{1} z_{0}\right]$ and $\mu=\left\langle\ldots w_{2} w_{1} w_{0}\right]$ be left rays such that $\operatorname{span}(\lambda) \subseteq \operatorname{dom}(\phi)$. We say that $\phi$ maps $\lambda$ onto $\mu$ if $z_{i} \phi=w_{i}$ for all $i \geq 0$. 


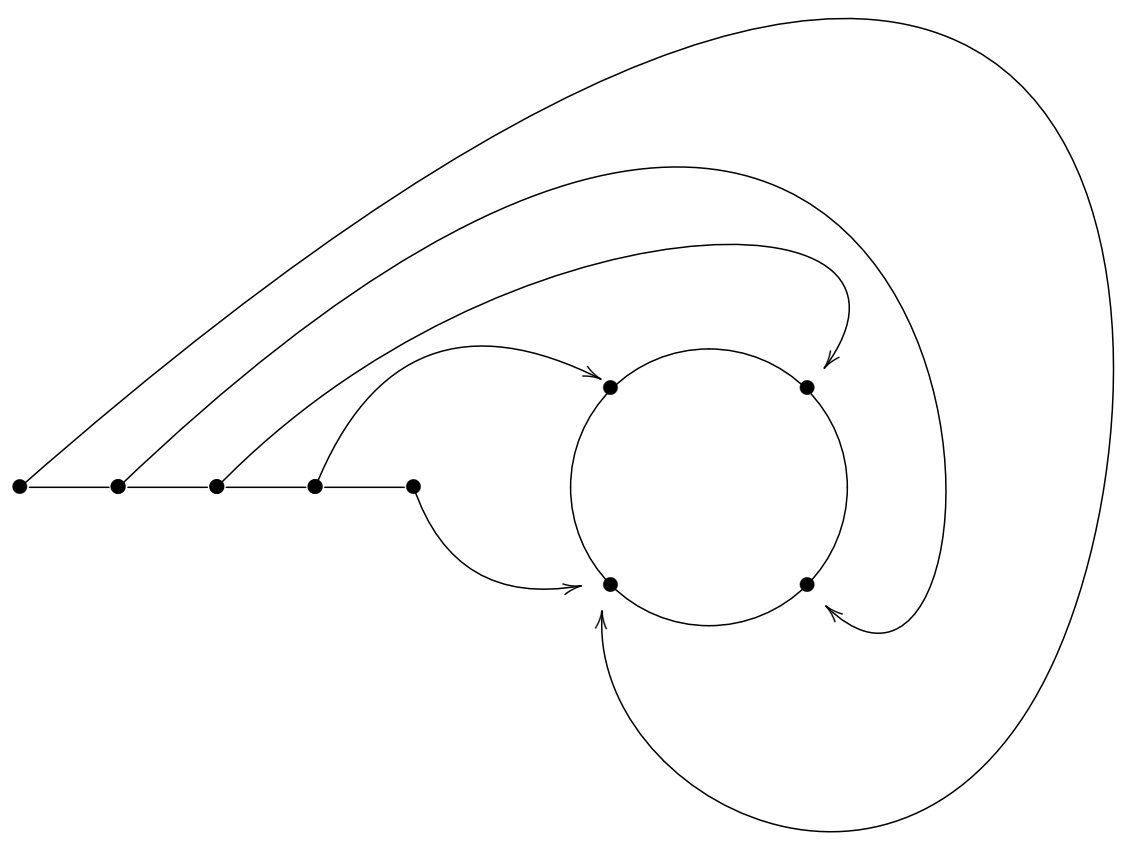

Figure 3.2: A chain wrapped around a cycle.

- Let $\omega=\left\langle\ldots x_{-1} x_{0} x_{1} \ldots\right\rangle$ and $\pi=\left\langle\ldots y_{-1} y_{0} y_{1} \ldots\right\rangle$ be double rays such that $\operatorname{dom}(\omega) \subseteq$ $\operatorname{dom}(\phi)$. We say that $\phi$ maps $\omega$ onto $\pi$ at $y_{t}$ if $x_{i} \phi=y_{t+i}$ for all $i$.

- Let $\eta=\left[x_{0} x_{1} x_{3} \ldots\right\rangle$ and $\mu=\left[y_{0} y_{1} y_{3} \ldots\right\rangle$ be right rays such that $\operatorname{dom}(\eta) \subseteq \operatorname{dom}(\phi)$. We say that $\phi$ maps $\eta$ onto $\mu$ if $x_{i} \phi=y_{i}$ for all $i \geq 0$.

We begin with the case when $\gamma$ has a cycle. If $D(\gamma)$ is homomorphic to $D(\delta)$, then $\delta$ must also have a cycle.

Lemma 3.4. Let $\gamma$ and $\delta$ be connected components of $\alpha \in T(X)$ such that $\gamma$ has a cycle $\sigma=$ $\left(x_{0} \ldots x_{k-1}\right)$. Let $\phi: \operatorname{dom}(\gamma) \rightarrow \operatorname{dom}(\delta)$. Then $\phi$ is a homomorphism from $D(\gamma)$ to $D(\delta)$ if and only if $\delta$ has a cycle $\theta=\left(y_{0} \ldots y_{m-1}\right)$ and the following conditions are satisfied:

(1) $m$ divides $k$;

(2) $\phi$ wraps $\sigma$ around $\theta$ at some $y_{t}$;

(3) If $\tau=\left[z_{0} \ldots z_{p}=x_{i}\right]$ is a finite branch of $\sigma$, then exactly one of the following holds:

(a) $\phi$ wraps $\tau$ around $\theta$ at $y_{t+i-p}$; or

(b) There is a branch $\mu=\left(\ldots w_{q} \ldots w_{0}=y_{j}\right]$ of $\theta$ with $1 \leq q \leq p$ and $j+p-q \equiv t+i$ $(\bmod m)$ such that $\phi$ maps $\left[z_{0} \ldots z_{q}\right]$ onto $\left[w_{q} \ldots w_{0}=y_{j}\right]$ and if $q<p$ then $\phi$ wraps $\left[z_{q} \ldots z_{p}=x_{i}\right]$ around $\theta$ at $y_{j}$;

(4) If $\lambda=\left\langle\ldots z_{2} z_{1} z_{0}=x_{i}\right]$ is an infinite branch of $\sigma$, then exactly one of the following holds: 


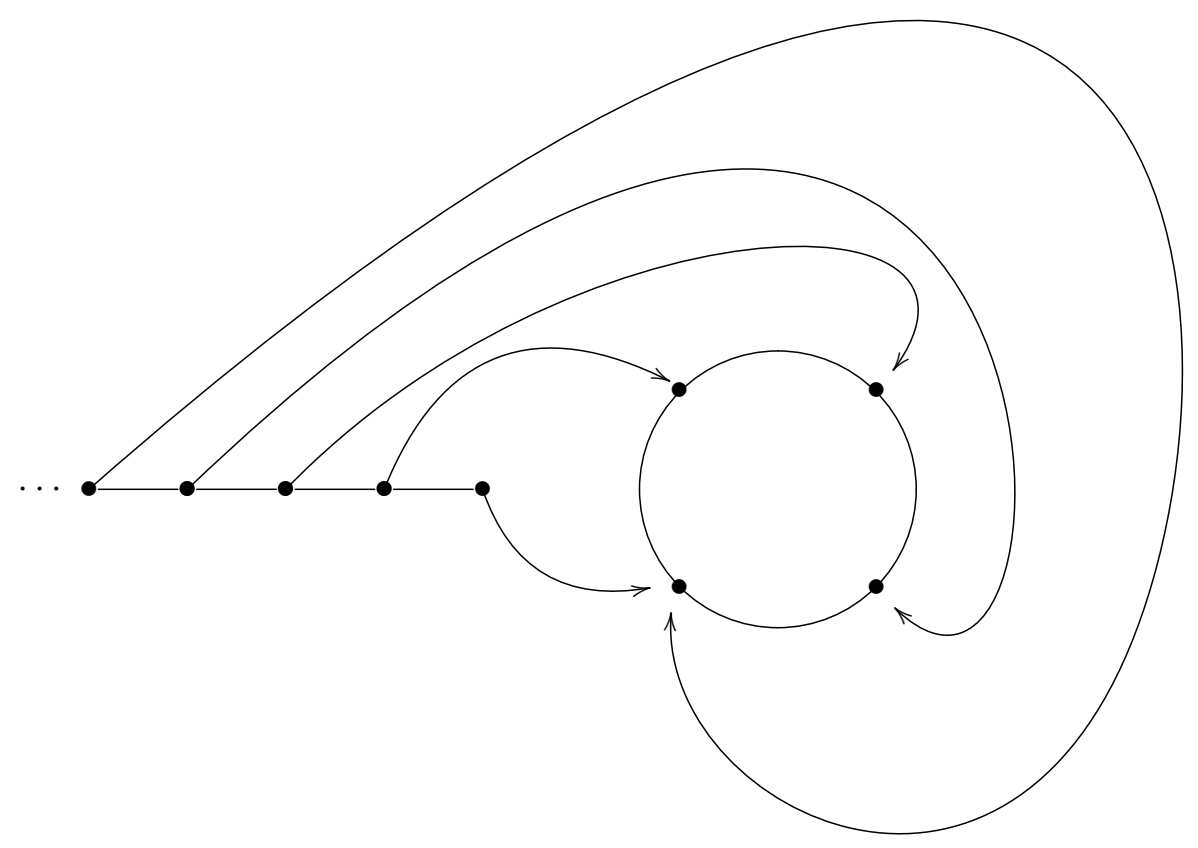

Figure 3.3: A left ray counter-wrapped around a cycle.

(a) $\phi$ counter-wraps $\lambda$ around $\theta$ at $y_{t+i}$; or

(b) There is an infinite branch $\mu=\left\langle\ldots w_{2} w_{1} w_{0}=y_{j}\right]$ of $\theta$ and there is $s \geq 0$ with $j+s \equiv t+i(\bmod m)$ such that $\phi$ maps $\left\langle\ldots z_{s+2} z_{s+1} z_{s}\right]$ onto $\mu$ and if $s>0$ then $\phi$ wraps $\left[z_{s} \ldots z_{1} z_{0}=x_{i}\right]$ around $\theta$ at $y_{j}$.

Proof. Suppose $\phi$ is a homomorphism from $D(\gamma)$ to $D(\delta)$. Since $\sigma$ is a cycle, we have

$$
x_{0} \rightarrow x_{1} \rightarrow \cdots \rightarrow x_{k-1} \rightarrow x_{0},
$$

in $D(\gamma)$, and so, since $\phi$ preserves edges,

$$
x_{0} \phi \rightarrow x_{1} \phi \rightarrow \cdots \rightarrow x_{k-1} \phi \rightarrow x_{0} \phi
$$

in $D(\delta)$. If $x_{0} \phi, x_{1} \phi, \ldots, x_{k-1} \phi$ are pairwise distinct, then $\theta=\left(x_{0} \phi x_{1} \phi \ldots x_{k-1} \phi\right)$ is a cycle in $\delta$ of length $k$ and $\phi$ wraps $\sigma$ around $\theta$ at $x_{0} \phi$. Otherwise, let $s$ be the smallest element of $\{0, \ldots, k-1\}$ such that $x_{s} \phi=x_{j} \phi$ for some $j \in\{s+1, \ldots, k-1\}$, and let $m$ be the smallest positive integer such that $x_{s} \phi=x_{s+m} \phi$. Then $\theta=\left(x_{s} \phi x_{s+1} \phi \ldots x_{s+m-1} \phi\right)$ is a cycle in $\delta$. Moreover, since (3.1) can be rewritten as

$$
x_{s} \phi \rightarrow x_{s+1} \phi \rightarrow \cdots \rightarrow x_{s+k-1} \phi \rightarrow x_{s} \phi,
$$

it follows that $m$ divides $k$ and $\phi$ wraps $\sigma$ around $\theta$ at some $x_{s+t} \phi$, where $0 \leq t<m$. We have proved that $\delta$ has a cycle $\theta=\left(y_{0} \ldots y_{m-1}\right)$ and that (1) and (2) are satisfied.

To prove (3), let $\tau=\left[z_{0} \ldots z_{p}=x_{i}\right]$ be a finite branch of $\sigma$. Since $x_{0} \phi=y_{t}$ and $\phi$ wraps $\sigma$ around $\theta$, we have $x_{i} \phi=y_{t+i}$. Suppose $z_{0} \phi=y_{j}$ lies on $\theta$. Then

$$
z_{0} \phi=y_{j} \rightarrow z_{1} \phi=y_{j+1} \rightarrow \cdots \rightarrow z_{p} \phi=y_{j+p} .
$$




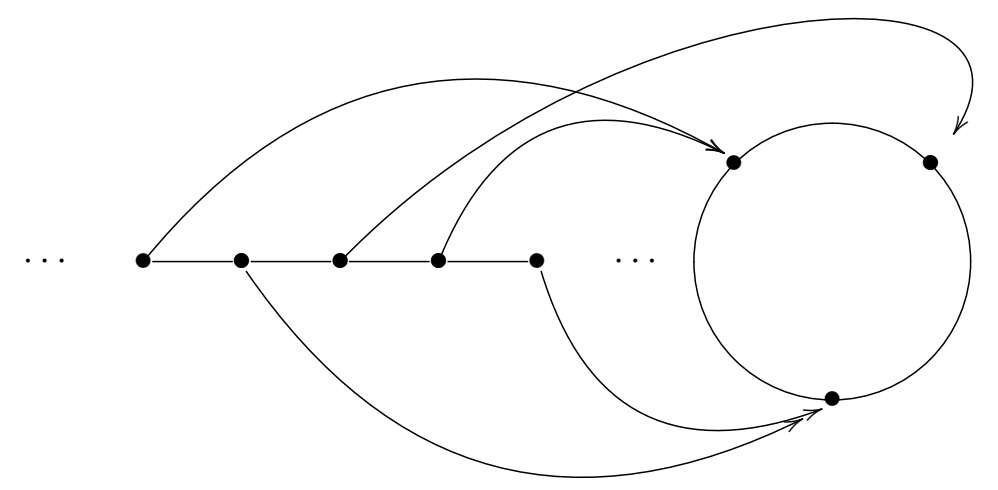

Figure 3.4: A double ray double-wrapped around a cycle.

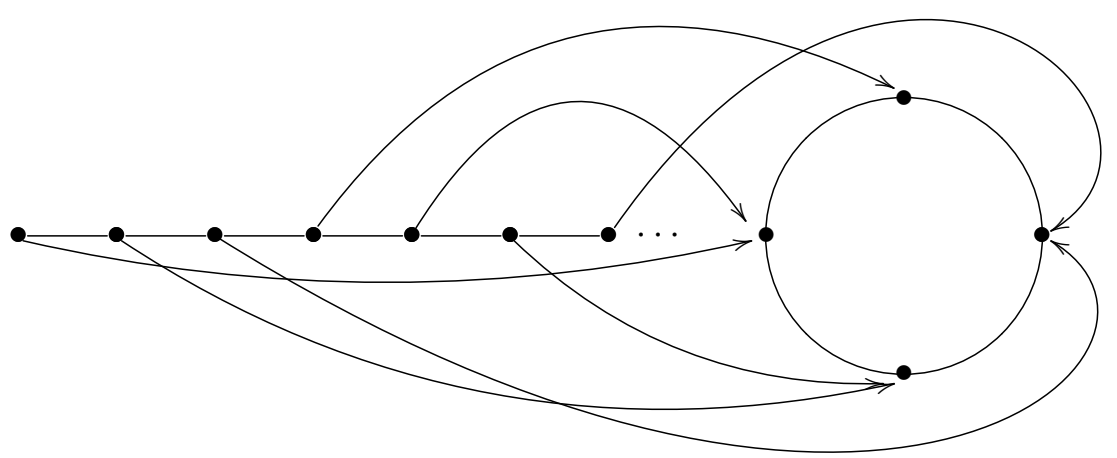

Figure 3.5: A right ray wrapped around a cycle.

Since $y_{j+p}=z_{p} \phi=x_{i} \phi=y_{t+i}$, we have $j+p \equiv t+i(\bmod m)$. Thus $y_{j}=y_{t+i-p}$, and so $\phi$ wraps $\tau$ around $\theta$ at $y_{t+i-p}$.

Suppose $z_{0} \phi$ does not lie on $\theta$. Then, by Proposition 2.13, $z_{0} \phi$ lies on some branch (finite or infinite) $\mu=\left(\ldots w_{2} w_{1} w_{0}=y_{j}\right]$ of $\theta$. Let $z_{0} \phi=w_{q}$. Then $q \geq 1\left(\right.$ since $\left.z_{0} \phi \notin \operatorname{dom}(\theta)\right)$ and

$$
z_{0} \phi=w_{q} \rightarrow z_{1} \phi=w_{q-1} \rightarrow \cdots \rightarrow z_{q} \phi=w_{0}=y_{j} \rightarrow z_{q+1} \phi=y_{j+1} \rightarrow \cdots \rightarrow z_{p} \phi=y_{j+p-q} .
$$

Thus $q \leq p, \phi$ maps $\left[z_{0} \ldots z_{q}\right]$ onto $\left[w_{q} \ldots w_{0}=y_{j}\right]$, and if $q<p$ then $\phi$ wraps $\left[z_{q} \ldots z_{p}=x_{i}\right]$ around $\theta$ at $y_{j}$. Further, $y_{j+p-q}=z_{p} \phi=x_{i} \phi=y_{t+i}$, and so $j+p-q \equiv t+i(\bmod m)$.

Hence (3a) or (3b) holds. Thus exactly one of them holds since (3a) and (3b) are mutually exclusive. We have proved (3). The proof of (4) is similar.

Conversely, suppose (1)-(4) are satisfied. Let $x \rightarrow z$ be an edge in $D(\gamma)$. If $x \in \operatorname{dom}(\sigma)$, then $x_{i}=x \rightarrow z=x_{i+1}$ for some $i$, and so $x \phi=x_{i} \phi=y_{t+i} \rightarrow y_{t+i+1}=x_{i+1} \phi=z \phi$ by (2). If $x \notin \operatorname{dom}(\sigma)$, then $x \rightarrow z$ is an edge of some branch of $\sigma$ (by Proposition 2.13), and so $x \phi \rightarrow z \phi$ in $D(\delta)$ by (3) and (4).

Figure 3.6 illustrates 3(b) of Lemma 3.4. Extending the finite branches in Figure 3.6 to infinite branches of the cycles, we obtain an illustration of 4(b) of Lemma 3.4.

Suppose $\gamma$ has a double ray and $D(\gamma)$ is homomorphic to $D(\delta)$. Then the situation is more complicated since $\delta$ may have either a cycle or a double ray. 


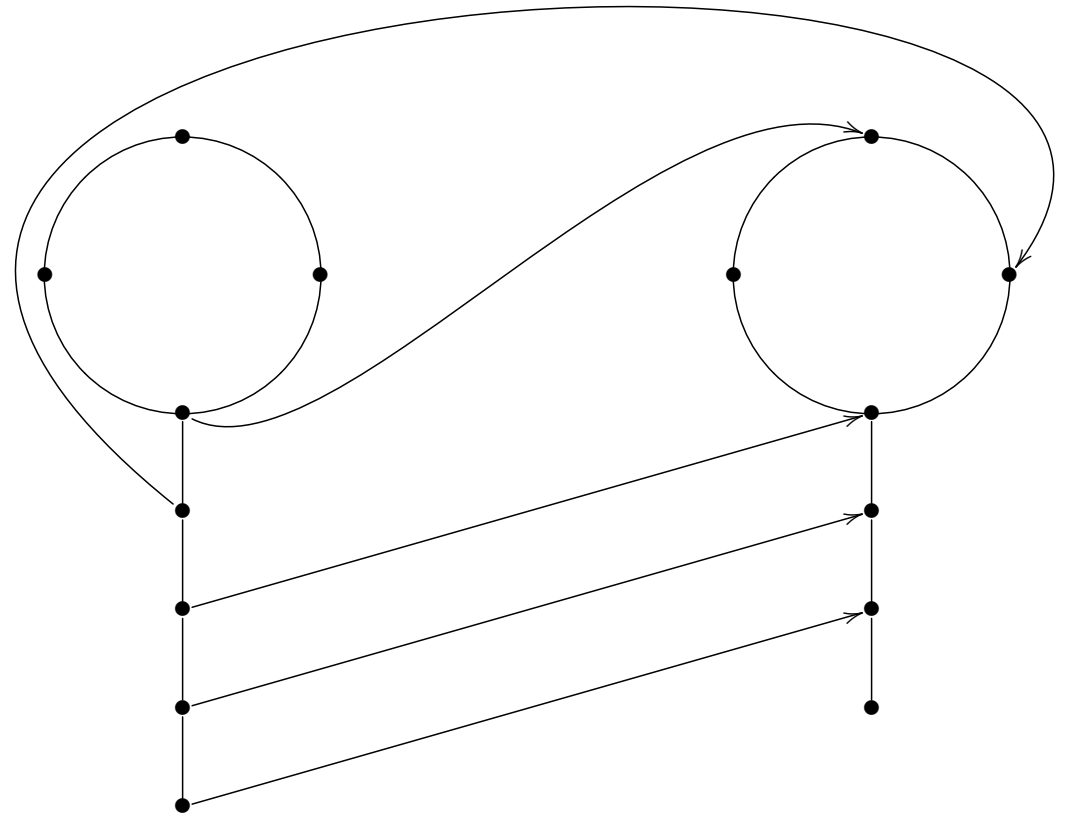

Figure 3.6: A homomorphism acting on a finite branch of a cycle in Lemma 3.4.

Lemma 3.5. Let $\gamma$ and $\delta$ be connected components of $\alpha \in T(X)$ such that $\gamma$ has a double ray $\omega=\left\langle\ldots x_{-1} x_{0} x_{1} \ldots\right\rangle$. Suppose $\phi$ is a homomorphism from $D(\gamma)$ to $D(\delta)$. Then exactly one of the following holds:

(a) $\delta$ has a cycle $\theta=\left(y_{0} \ldots y_{m-1}\right)$ and either $\phi$ double wraps $\omega$ around $\theta$ at some $y_{t}$ or there is $u$ such that $\phi$ wraps $\left[x_{u} x_{u+1} x_{u+2} \ldots\right\rangle$ around $\theta$ at some $y_{t}$ and $x_{u-1} \notin \operatorname{dom}(\theta)$; or

(b) $\delta$ has a double ray $\pi$ such that $\phi$ maps $\omega$ onto $\pi$.

Proof. Since $\phi$ preserves edges, we have

$$
\cdots \rightarrow x_{-1} \phi \rightarrow x_{0} \phi \rightarrow x_{1} \phi \rightarrow \cdots .
$$

Suppose $\delta$ contains a cycle $\theta=\left(y_{0} \ldots y_{m-1}\right)$. Since $x_{0} \phi, y_{0} \in \operatorname{dom}(\delta)$ and $\delta$ is a connected component of $\alpha$, there are integers $p, q \geq 0$ such that $\left(x_{0} \phi\right) \alpha^{p}=y_{0} \alpha^{q}$. By (3.2), $\left(x_{0} \phi\right) \alpha^{p}=x_{p} \phi$, and so $x_{p} \phi$ lies on $\theta$. Suppose every $x_{i} \phi$ lies on $\theta$ and let $x_{0} \phi=y_{t}$. Then $\phi$ double-wraps $\omega$ around $\theta$ at $y_{t}$ by (3.2). Suppose not every $x_{i} \phi$ lies on $\theta$. Since $x_{p} \phi$ lies on $\theta$, there is $u$ such that $x_{u} \phi$ lies on $\theta$ but $x_{u-1} \phi$ does not. Let $x_{u} \phi=y_{t}$. Then, by (3.2), $\phi$ wraps $\left[x_{u} x_{u+1} x_{u+2} \ldots\right\rangle$ around $\theta$ at $y_{t}$ and $x_{u-1} \notin \operatorname{dom}(\theta)$.

Suppose $\delta$ does not contain a cycle. Then the vertices in (3.2) must be pairwise distinct. Thus $\pi=\left\langle\ldots x_{-1} \phi x_{0} \phi x_{1} \phi \ldots\right\rangle$ is a double ray in $\delta$ and $\phi$ maps $\omega$ onto $\pi$.

We have proved that (a) or (b) holds. By Proposition 2.10, (a) and (b) are mutually exclusive, so exactly one of them holds.

We now analyze what happens in each of the cases exhibited by Lemma 3.5. 
Lemma 3.6. Let $\gamma$ and $\delta$ be connected components of $\alpha \in T(X)$ such that $\gamma$ has a double ray $\omega=\left\langle\ldots x_{-1} x_{0} x_{1} \ldots\right\rangle$ and $\delta$ has a cycle $\theta=\left(y_{0} \ldots y_{m-1}\right)$. Let $\phi: \operatorname{dom}(\gamma) \rightarrow \operatorname{dom}(\delta)$ be such that $\phi$ double wraps $\omega$ around $\theta$ at some $y_{t}$. Then $\phi$ is a homomorphism from $D(\gamma)$ to $D(\delta)$ if and only if the following conditions are satisfied:

(1) If $\tau=\left[z_{0} \ldots z_{p}=x_{i}\right]$ is a finite branch of $\omega$, then exactly one of the following holds:

(a) $\phi$ wraps $\tau$ around $\theta$ at $y_{t+i-p}$; or

(b) There is a branch $\mu=\left(\ldots w_{q} \ldots w_{0}=y_{j}\right]$ of $\theta$ with $1 \leq q \leq p$ and $j+p-q \equiv t+i$ $(\bmod m)$ such that $\phi$ maps $\left[z_{0} \ldots z_{q}\right]$ onto $\left[w_{q} \ldots w_{0}=y_{j}\right]$ and if $q<p$ then $\phi$ wraps $\left[z_{q} \ldots z_{p}=x_{i}\right]$ around $\theta$ at $y_{j}$;

(2) If $\lambda=\left\langle\ldots z_{2} z_{1} z_{0}=x_{i}\right]$ is an infinite branch of $\omega$, then exactly one of the following holds:

(a) $\phi$ counter-wraps $\lambda$ around $\theta$ at $y_{t+i}$; or

(b) There is an infinite branch $\mu=\left\langle\ldots w_{2} w_{1} w_{0}=y_{j}\right]$ of $\theta$ and there is $s \geq 0$ with $j+s \equiv t+i(\bmod m)$ such that $\phi$ maps $\left\langle\ldots z_{s+2} z_{s+1} z_{s}\right]$ onto $\mu$ and if $s>0$ then $\phi$ wraps $\left[z_{s} \ldots z_{0}=x_{i}\right]$ around $\theta$ at $y_{j}$.

Proof. Suppose $\phi$ is a homomorphism from $D(\gamma)$ to $D(\delta)$. To prove (1), follow the proof of Lemma 3.4(3) (almost verbatim). The proof of (2) is similar.

Conversely, suppose (1) and (2) are satisfied. Let $x \rightarrow z$ be an edge in $D(\gamma)$. If $x \in \operatorname{dom}(\omega)$, then $x_{i}=x \rightarrow z=x_{i+1}$ for some $i$, and so $x \phi=x_{i} \phi=y_{t+i} \rightarrow y_{t+i+1}=x_{i+1} \phi=z \phi$ in $D(\delta)$ since $\phi$ double-wraps $\omega$ around $\theta$ at $y_{t}$. If $x \notin \operatorname{dom}(\omega)$, then $x \rightarrow z$ is an edge of some branch of $\omega$ (by Proposition 2.13), and so $x \phi \rightarrow z \phi$ in $D(\delta)$ by (1) and (2).

Lemma 3.7. Let $\gamma$ and $\delta$ be connected components of $\alpha \in T(X)$ such that $\gamma$ has a double ray $\omega=\left\langle\ldots x_{-1} x_{0} x_{1} \ldots\right\rangle$ and $\delta$ has a cycle $\theta=\left(y_{0} \ldots y_{m-1}\right)$. Let $\phi: \operatorname{dom}(\gamma) \rightarrow \operatorname{dom}(\delta)$ be such that for some $u$, $\phi$ wraps $\left[x_{u} x_{u+1} x_{u+2} \ldots\right\rangle$ around $\theta$ at some $y_{t}$ and $x_{u-1} \notin \operatorname{dom}(\theta)$. Then $\phi$ is a homomorphism from $D(\gamma)$ to $D(\delta)$ if and only if the following conditions are satisfied:

(1) There is an infinite branch $\mu=\left\langle\ldots v_{2} v_{1} v_{0}=y_{t}\right]$ of $\theta$ such that $\phi$ maps $\left\langle\ldots x_{u-2} x_{u-1} x_{u}\right]$ onto $\mu$.

(2) If $\tau=\left[z_{0} \ldots z_{p}=x_{i}\right]$ is a finite branch of $\omega$ with $i \geq u$, then exactly one of the following holds:

(a) $\phi$ wraps $\tau$ around $\theta$ at $y_{t+i-u-p}$; or

(b) There is a branch $\left(\ldots w_{q} \ldots w_{0}=y_{j}\right]$ of $\theta$ with $1 \leq q \leq p$ and $j+p-q \equiv t+i-u$ $(\bmod m)$ such that $\phi$ maps $\left[z_{0} \ldots z_{q}\right]$ onto $\left[w_{q} \ldots w_{0}=y_{j}\right]$ and if $q<p$ then $\phi$ wraps $\left[z_{q} \ldots z_{p}=x_{i}\right]$ around $\theta$ at $y_{j}$;

(3) If $\tau=\left[z_{0} \ldots z_{p}=x_{i}\right]$ is a finite branch of $\omega$ with $i<u$, then exactly one of the following holds:

(a) $\phi$ maps $\tau$ onto $\left[v_{u-i-p} \ldots v_{u-i}\right]$; or

(b) There is a branch $\left(\ldots w_{q} \ldots w_{1} w_{0}=v_{j} \ldots v_{0}=y_{t}\right]$ of $\theta$ with $1 \leq q \leq p, j+p-q=$ $u-i$, and $w_{1} \notin \operatorname{dom}(\mu)$ such that $\phi$ maps $\left[z_{0} \ldots z_{q}\right]$ onto $\left[w_{q} \ldots w_{0}=v_{j}\right]$ and if $q<p$ then $\phi$ maps $\left[z_{q} \ldots z_{p}=x_{i}\right]$ onto $\left[v_{j} \ldots v_{u-i}\right]$; 
(4) If $\lambda=\left\langle\ldots z_{2} z_{1} z_{0}=x_{i}\right]$ is an infinite branch of $\omega$ with $i \geq u$, then exactly one of the following holds:

(a) $\phi$ counter-wraps $\lambda$ around $\theta$ at $y_{t+i-u}$; or

(b) There is an infinite branch $\mu_{1}=\left\langle\ldots y_{j}\right]$ of $\theta$ and there is $s \geq 0$ with $j+s \equiv t+$ $i-u(\bmod m)$ such that $\phi$ maps $\left\langle\ldots z_{s+2} z_{s+1} z_{s}\right]$ onto $\mu_{1}$ and if $s>0$ then $\phi$ wraps $\left[z_{s} \ldots z_{0}=x_{i}\right]$ around $\theta$ at $y_{j}$;

(5) If $\lambda=\left\langle\ldots z_{2} z_{1} z_{0}=x_{i}\right]$ is an infinite branch of $\omega$ with $i<u$, then exactly one of the following holds:

(a) $\phi$ maps $\lambda$ onto $\left\langle\ldots v_{u-i-2} v_{u-i-1} v_{u-i}\right]$; or

(b) There is an infinite branch $\left\langle\ldots w_{2} w_{1} w_{0}=v_{j} \ldots v_{0}=y_{t}\right]$ of $\theta$ such that $w_{1}$ does not lie on $\mu$, and there is $s \geq 0$ with $j+s=u-i$ such that $\phi$ maps $\left\langle\ldots z_{s+2} z_{s+1} z_{s}\right]$ onto $\left\langle\ldots w_{2} w_{1} w_{0}=v_{j}\right]$ and if $s>0$ then $\phi$ maps $\left[z_{s} \ldots z_{0}=x_{i}\right]$ onto $\left[v_{j} \ldots v_{u-i}\right]$.

Proof. Suppose $\phi$ is a homomorphism from $D(\gamma)$ to $D(\delta)$. Then, since $\phi$ wraps $\left[x_{u} x_{u+1} x_{u+2} \ldots\right\rangle$ around $\theta$ at $y_{t}$, we have

$$
\cdots \rightarrow x_{u-2} \phi \rightarrow x_{u-1} \phi \rightarrow x_{u} \phi=y_{t} \rightarrow x_{u+1} \phi=y_{t+1} \rightarrow \cdots .
$$

Thus, since $x_{u-1} \phi \notin \operatorname{dom}(\theta), \mu=\left\langle\ldots x_{u-2} \phi x_{u-1} \phi x_{u} \phi=y_{t}\right]$ is an infinite branch of $\theta$ and $\phi$ maps $\left\langle\ldots x_{u-2} x_{u-1} x_{u}\right]$ onto $\mu$. We have proved (1).

To prove (2), we follow the proof of Lemma 3.4(3). Let $\tau=\left[z_{0} \ldots z_{p}=x_{i}\right]$ be a finite branch of $\omega$ with $i \geq u$. Since $x_{u} \phi=y_{t}$ and $\phi$ wraps $\left[x_{u} x_{u+1} x_{u+2} \ldots\right\rangle$ around $\theta$, we have $x_{i} \phi=y_{t+i-u}$. Suppose $z_{0} \phi=y_{j}$ lies on $\theta$. Then

$$
z_{0} \phi=y_{j} \rightarrow z_{1} \phi=y_{j+1} \rightarrow \cdots \rightarrow z_{p} \phi=y_{j+p}
$$

Since $y_{j+p}=x_{i} \phi=y_{t+i-u}$, we have $j+p \equiv t+i-u(\bmod m)$. Thus $y_{j}=y_{t+i-u-p}$, and so $\phi$ wraps $\tau$ around $\theta$ at $y_{t+i-u-p}$.

Suppose $z_{0} \phi$ does not lie on $\theta$. Then, by Proposition 2.13, $z_{0} \phi$ lies on some branch $\mu=$ $\left(\ldots w_{2} w_{1} w_{0}=y_{j}\right]$ of $\theta$. Let $z_{0} \phi=w_{q}$. Then $q \geq 1$ (since $\left.z_{0} \phi \notin \operatorname{dom}(\theta)\right)$ and

$$
z_{0} \phi=w_{q} \rightarrow z_{1} \phi=w_{q-1} \rightarrow \cdots \rightarrow z_{q} \phi=w_{0}=y_{j} \rightarrow z_{q+1} \phi=y_{j+1} \rightarrow \cdots \rightarrow z_{p} \phi=y_{j+p-q} .
$$

Thus $q \leq p, \phi$ maps $\left[z_{0} \ldots z_{q}\right]$ onto $\left[w_{q} \ldots w_{0}=y_{j}\right]$, and if $q<p$ then $\phi$ wraps $\left[z_{q} \ldots z_{p}=x_{i}\right]$ around $\theta$ at $y_{j}$. Further, $y_{j+p-q}=z_{p} \phi=x_{i} \phi=y_{t+i-u}$, and so $j+p-q \equiv t+i-u(\bmod m)$.

Hence (2a) or (2b) holds. Thus exactly one of them holds since (2a) and (2b) are mutually exclusive. We have proved (2). The proofs of (3)-(5) are similar.

Conversely, suppose (1)-(5) are satisfied. Then $\phi$ is a homomorphism from $D(\gamma)$ to $D(\delta)$ by an argument similar to the one used in the proof of Lemma 3.6.

Lemma 3.8. Let $\gamma$ and $\delta$ be connected components of $\alpha \in T(X)$ such that $\gamma$ has a double ray $\omega=\left\langle\ldots x_{-1} x_{0} x_{1} \ldots\right\rangle$ and $\delta$ has a double ray $\pi=\left\langle\ldots y_{-1} y_{0} y_{1} \ldots\right\rangle$. Let $\phi: \operatorname{dom}(\gamma) \rightarrow \operatorname{dom}(\delta)$ be such that $\phi$ maps $\omega$ onto $\pi$ at some $y_{t}$. Then $\phi$ is a homomorphism from $D(\gamma)$ to $D(\delta)$ if and only if the following conditions are satisfied:

(1) If $\tau=\left[z_{0} \ldots z_{p}=x_{i}\right]$ is a finite branch of $\omega$, then exactly one of the following holds: 
(a) $\phi$ maps $\tau$ onto $\left[y_{t+i-p} \ldots y_{t+i}\right]$; or

(b) There is a branch $\mu=\left(\ldots w_{q} \ldots w_{0}=y_{t+i-p+q}\right]$ of $\pi$ with $1 \leq q \leq p$ such that $\phi$ maps $\left[z_{0} \ldots z_{q}\right]$ onto $\left[w_{q} \ldots w_{0}=y_{t+i-p+q}\right]$ and if $q<p$ then $\phi$ maps $\left[z_{q} \ldots z_{p}=x_{i}\right]$ onto $\left[y_{t+i-p+q} \ldots y_{t+i}\right]$;

(2) If $\lambda=\left\langle\ldots z_{2} z_{1} z_{0}=x_{i}\right]$ is an infinite branch of $\omega$, then exactly one of the following holds:

(a) $\phi$ maps $\lambda$ onto $\left\langle\ldots y_{t+i-2} y_{t+i-1} y_{t+i}\right]$; or

(b) There is an infinite branch $\mu=\left\langle\ldots w_{2} w_{1} w_{0}=y_{t+i-s}\right]$ of $\pi$ with $s \geq 0$ such that $\phi$ maps $\left\langle\ldots z_{s+2} z_{s+1} z_{s}\right]$ onto $\mu$ and if $s>0$ then $\phi$ maps $\left[z_{s} \ldots z_{0}=x_{i}\right]$ onto $\left[y_{t+i-s} \ldots y_{t+i}\right]$.

Proof. Suppose $\phi$ is a homomorphism from $D(\gamma)$ to $D(\delta)$. Let $\tau=\left[z_{0} \ldots z_{p}=x_{i}\right]$ be a finite branch of $\omega$. Since $\phi$ preserves edges, we have

$$
z_{0} \phi \rightarrow z_{1} \phi \rightarrow \cdots \rightarrow z_{p} \phi=x_{i} \phi=y_{t+i}
$$

where the last equality is true since $x_{0} \phi=y_{t}$ and $\phi$ maps $\omega$ onto $\pi$. Suppose $z_{0} \phi$ lies on $\pi$. Then, by (3.3), $z_{0} \phi=y_{t+i-p}$ and $\phi$ maps $\tau$ onto $\left[y_{t+i-p} \ldots y_{t+i}\right]$.

Suppose $z_{0} \phi$ does not lie on $\pi$. Then, by Proposition $2.13, z_{0} \phi$ lies on some branch $\mu=$ $\left(\ldots w_{2} w_{1} w_{0}=y_{j}\right]$ of $\theta$. Let $z_{0} \phi=w_{q}$. Then $q \geq 1$ (since $z_{0} \phi$ does not lie on $\pi$ ) and

$$
z_{0} \phi=w_{q} \rightarrow \cdots \rightarrow z_{q} \phi=w_{0}=y_{j} \rightarrow z_{q+1} \phi=y_{j+1} \rightarrow \cdots \rightarrow z_{p} \phi=y_{j+p-q} .
$$

Since $y_{j+p-q}=z_{p} \phi=x_{i} \phi=y_{t+i}$, we have $j=t+i-p+q$. Thus, by (3.4), $\phi$ maps $\left[z_{0} \ldots z_{q}\right]$ onto $\left[w_{q} \ldots w_{0}=y_{t+i-p+q}\right]$, and if $q<p$ then $\phi$ maps $\left[z_{q} \ldots z_{p}=x_{i}\right]$ onto $\left[y_{t+i-p+q} \ldots y_{t+i}\right]$. Hence (1a) or (1b) holds. Thus exactly one of them holds since (1a) and (1b) are mutually exclusive. We have proved (1). The proof of (2) is similar.

Conversely, suppose (1) and (2) are satisfied. Then $\phi$ is a homomorphism from $D(\gamma)$ to $D(\delta)$ by an argument similar to the one used in the proof of Lemma 3.6.

Figure 3.7 illustrates 2(b) of Lemma 3.8, where the assumption is that $\omega=\left\langle\ldots x_{-1} x_{0} x_{1} \ldots\right\rangle$ and $\pi=\left\langle\ldots y_{-1} y_{0} y_{1} \ldots\right\rangle$ are vertical double rays in the figure.

Finally, if $\gamma$ is of type rro (see Definition 2.11) and $D(\gamma)$ is homomorphic to $D(\delta)$, then $\delta$ may have a cycle, or a double ray, or be of type rro.

Lemma 3.9. Let $\gamma$ and $\delta$ be connected components of $\alpha \in T(X)$ such that $\gamma$ is of type rro, and let $\eta=\left[x_{0} x_{1} x_{2} \ldots\right\rangle$ be a maximal right ray in $\gamma$. Suppose $\phi$ is a homomorphism from $D(\gamma)$ to $D(\delta)$. Then exactly one of the following holds:

(a) $\delta$ has a cycle $\theta=\left(y_{0} \ldots y_{m-1}\right)$ and either $\phi$ wraps $\eta$ around $\theta$ at some $y_{t}$, or there is $u \geq 1$ such that $\phi$ wraps $\left[x_{u} x_{u+1} x_{u+2} \ldots\right\rangle$ around $\theta$ at some $y_{t}$ and $x_{u-1} \notin \operatorname{dom}(\theta)$;

(b) $\delta$ has a double ray $\pi=\left\langle\ldots y_{-1} y_{0} y_{1} \ldots\right\rangle$ such that either $\phi$ maps $\eta$ onto $\left[y_{t} y_{t+1} \ldots\right\rangle$ for some $t$, or there are $t$ and $u \geq 1$ such that $\phi$ maps $\left[x_{u} x_{u+1} \ldots\right\rangle$ onto $\left[y_{t} y_{t+1} \ldots\right\rangle, x_{u-1} \notin$ $\operatorname{dom}(\pi)$, and $x_{0}$ does not lie on an infinite branch of $\pi ;$ or

(c) $\delta$ is of type rro and has a maximal right ray $\mu=\left[y_{0} y_{1} y_{2} \ldots\right]$ such that $\phi$ maps $\eta$ onto $\left[y_{t} y_{t+1} \ldots\right\rangle$ for some $t \geq 0$. 


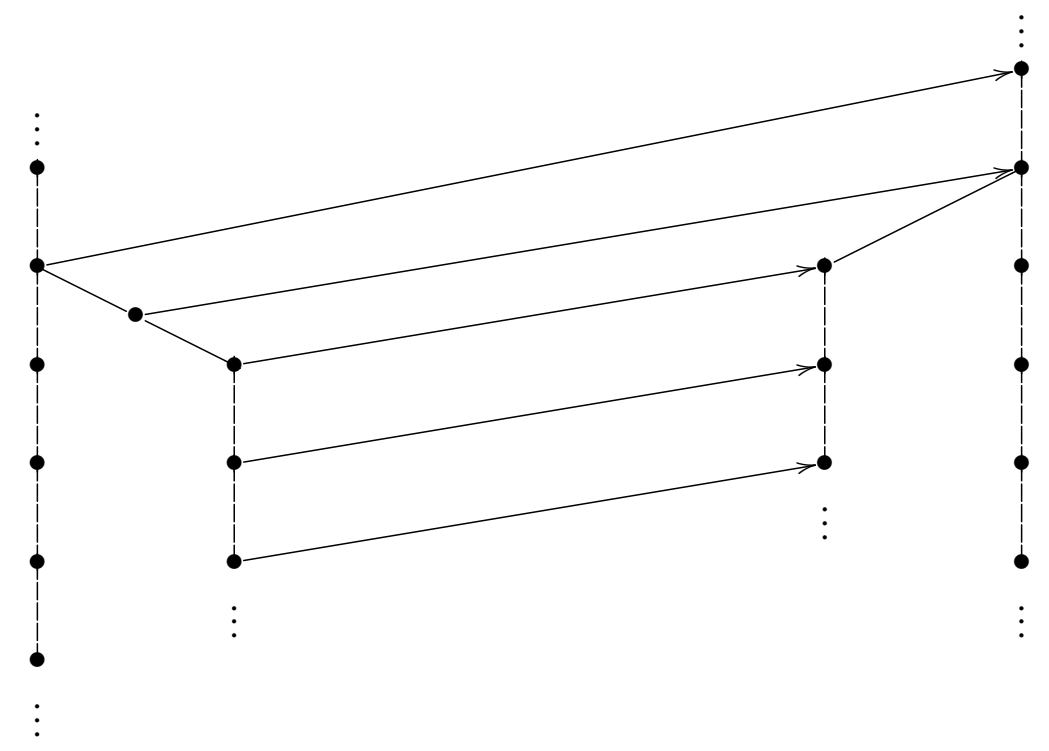

Figure 3.7: A homomorphism acting on an infinite branch of a double ray in Lemma 3.8.

Proof. Since $\phi$ preserves edges, we have

$$
x_{0} \phi \rightarrow x_{1} \phi \rightarrow x_{2} \phi \rightarrow \cdots .
$$

If $\delta$ contains a cycle $\theta=\left(y_{0} \ldots y_{m-1}\right)$, then (a) holds by an argument similar to the proof of Lemma 3.5(a).

Suppose $\delta$ contains a double ray. Then the vertices in (3.5) must be pairwise distinct since otherwise $\delta$ would also contain a cycle, which is impossible by Proposition 2.10. Suppose there are vertices $w_{0}, w_{1}, w_{2}, \ldots$ of $D(\delta)$ such that

$$
\cdots \rightarrow w_{2} \rightarrow w_{1} \rightarrow w_{0} \rightarrow x_{0} \phi \rightarrow x_{1} \phi \rightarrow x_{2} \phi \rightarrow \cdots .
$$

Then $\delta$ has a double ray $\pi=\left\langle\ldots y_{-1} y_{0} y_{1} \ldots\right\rangle$, namely $\pi=\left\langle\ldots w_{1} w_{0} x_{0} \phi x_{1} \phi \ldots\right\rangle$ such that $\phi$ maps $\eta$ onto $\left[y_{t} y_{t+1} \ldots\right\rangle$ for some $t$. Suppose there are no vertices $w_{0}, w_{1}, w_{2}, \ldots$ of $D(\delta)$ such that (3.6) holds. Let $\pi=\left\langle\ldots y_{-1} y_{0} y_{1} \ldots\right\rangle$ be any double ray in $\delta$. Let $u$ be the smallest non-negative integer such that $x_{u} \phi=y_{t}$ for some $t$. (Such $u$ must exist since $\delta$ is a connected component of $\alpha$.) Then $u \geq 1$ since if $u=0$ then (3.6) would hold for $w_{0}=y_{t-1}, w_{1}=y_{t-2}, \ldots$ Moreover, $\phi$ maps $\left[x_{u} x_{u+1} \ldots\right\rangle$ onto $\left[y_{t} y_{t+1} \ldots\right\rangle$ (since $x_{u} \phi=y_{t}$ ), $x_{u-1} \notin \operatorname{dom}(\pi)$ (by the choice of $u$ ), and $x_{0} \phi$ does not lie on an infinite branch of $\pi$ (since if it did then (3.6) would hold for the vertices $w_{0}, w_{1}, \ldots$ preceding $x_{0} \phi$ on that branch).

Suppose $\delta$ does not contain a cycle or a double ray. Then $\delta$ is of type rro by Proposition 2.10. Let $w_{0}, \ldots, w_{q}, q \geq 0$, be vertices in $D(\delta)$ such that

$$
w_{0} \rightarrow \cdots \rightarrow w_{q}=x_{0} \phi \rightarrow x_{1} \phi \rightarrow x_{2} \phi \rightarrow \cdots
$$

and $w_{0} \notin \operatorname{im}(\alpha)$. (Such vertices must exist since otherwise $\gamma$ would have a double ray.) Then $\mu=\left[w_{0} \ldots w_{q}=x_{0} \phi x_{1} \phi x_{2} \phi \ldots\right\rangle$ is a desired maximal right ray in $\delta$ from (c).

We have proved that at least one of (a)-(c) holds. By Proposition 2.10, (a), (b), (c) are pairwise mutually exclusive, so exactly one of them holds. 
We now analyze what happens in each of the cases exhibited by Lemma 3.9. Recall that, by Proposition 2.13, if $\gamma$ is of type $\operatorname{rro}$ and $\eta$ is a maximal right ray in $\gamma$, then all branches of $\eta$ are finite.

Lemma 3.10. Let $\gamma$ and $\delta$ be connected components of $\alpha \in T(X)$ such that $\gamma$ is of type rro and has a maximal right ray $\eta=\left[x_{0} x_{1} x_{2} \ldots\right\rangle$, and $\delta$ has a cycle $\theta=\left(y_{0} \ldots y_{m-1}\right)$. Let $\phi: \operatorname{dom}(\gamma) \rightarrow$ $\operatorname{dom}(\delta)$ be such that $\phi$ wraps $\eta$ around $\theta$ at some $y_{t}$. Then $\phi$ is a homomorphism from $D(\gamma)$ to $D(\delta)$ if and only if for every (finite) branch $\tau=\left[z_{0} \ldots z_{p}=x_{i}\right]$ of $\eta$, exactly one of the following holds:

(a) $\phi$ wraps $\tau$ around $\theta$ at $y_{t+i-p}$; or

(b) There is a branch $\mu=\left(\ldots w_{q} \ldots w_{0}=y_{j}\right]$ of $\theta$ with $1 \leq q \leq p$ and $j+p-q \equiv t+i$ $(\bmod m)$ such that $\phi$ maps $\left[z_{0} \ldots z_{q}\right]$ onto $\left[w_{q} \ldots w_{0}=y_{j}\right]$ and if $q<p$ then $\phi$ wraps $\left[z_{q} \ldots z_{p}=x_{i}\right]$ around $\theta$ at $y_{j}$.

Proof. Similar to the proof of Lemma 3.7(2).

Lemma 3.11. Let $\gamma$ and $\delta$ be connected components of $\alpha \in T(X)$ such that $\gamma$ is of type rro and has a right ray $\eta=\left[x_{0} x_{1} x_{2} \ldots\right\rangle$, and $\delta$ has a cycle $\theta=\left(y_{0} \ldots y_{m-1}\right)$. Let $\phi: \operatorname{dom}(\gamma) \rightarrow \operatorname{dom}(\delta)$ be such that for some $u \geq 1, \phi$ wraps $\left[x_{u} x_{u+1} x_{u+2} \ldots\right\rangle$ around $\theta$ at some $y_{t}$ and $x_{u-1} \notin \operatorname{dom}(\theta)$. Then $\phi$ is a homomorphism from $D(\gamma)$ to $D(\delta)$ if and only if the following conditions are satisfied:

(1) There is a branch (finite or infinite) $\mu=\left(\ldots v_{2} v_{1} v_{0}=y_{t}\right]$ of $\theta$ such that the length of $\mu$ is at least $u+1$ and $\phi$ maps $\left[x_{0} \ldots x_{u}\right]$ onto $\left[v_{u} \ldots v_{0}\right]$.

(2) If $\tau=\left[z_{0} \ldots z_{p}=x_{i}\right]$ is a branch of $\eta$ with $i \geq u$, then exactly one of the following holds:

(a) $\phi$ wraps $\tau$ around $\theta$ at $y_{t+i-u-p}$; or

(b) There is a branch $\left(\ldots w_{q} \ldots w_{0}=y_{j}\right]$ of $\theta$ with $1 \leq q \leq p$ and $j+p-q \equiv t+i-u$ $(\bmod m)$ such that $\phi$ maps $\left[z_{0} \ldots z_{q}\right]$ onto $\left[w_{q} \ldots w_{q}=y_{j}\right]$ and if $q<p$ then $\phi$ wraps $\left[z_{q} \ldots z_{p}=x_{i}\right]$ around $\theta$ at $y_{j}$;

(3) If $\tau=\left[z_{0} \ldots z_{p}=x_{i}\right]$ is a branch of $\eta$ with $i<u$, then exactly one of the following holds:

(a) $\phi$ maps $\tau$ onto $\left[v_{u-i+p} \ldots v_{u-i}\right]$; or

(b) There is a branch $\left(\ldots w_{q} \ldots w_{1} w_{0}=v_{j} \ldots v_{0}=y_{t}\right]$ of $\theta$ with $1 \leq q \leq p, j-p+q=$ $u-i$ and $w_{1} \notin \operatorname{dom}(\mu)$ such that $\phi$ maps $\left[z_{0} \ldots z_{q}\right]$ onto $\left[w_{q} \ldots w_{0}=v_{j}\right]$ and if $q<p$ then $\phi$ maps $\left[z_{q} \ldots z_{p}=x_{i}\right]$ onto $\left[v_{j} \ldots v_{u-i}\right]$.

Proof. Suppose $\phi$ is a homomorphism from $D(\gamma)$ to $D(\delta)$. Then (1) and (2) follow by an argument similar to the proof of Lemma 3.7(1)(2).

To prove (3), let $\tau=\left[z_{0} \ldots z_{p}=x_{i}\right]$ be a branch of $\eta$ with $i<u$. Since $\phi$ maps $\left[x_{0} \ldots x_{u}\right]$ onto $\left[v_{u} \ldots v_{0}\right]$ (by (1)) and $z_{p}=x_{i}$ with $i<u$, we have $z_{p} \phi=x_{i} \phi=v_{u-i}$.

Suppose $z_{0} \phi \in \operatorname{dom}(\mu)$, that is, $z_{0} \phi=v_{s}$ for some $s \geq 1$. Then

$$
z_{0} \phi=v_{s} \rightarrow z_{1} \phi=v_{s-1} \rightarrow \cdots \rightarrow z_{p} \phi=v_{s-p} .
$$

We have $v_{s-p}=z_{p} \phi=v_{u-i}$, and so $s-p=u-i$. Thus $s=u-i+p$, and so $\phi$ maps $\tau$ onto $\left[v_{u-i+p} \ldots v_{u-i}\right]$ by (3.7). 
Suppose $z_{0} \phi \notin \operatorname{dom}(\mu)$. Let $q$ be the smallest integer in $\{1, \ldots, p\}$ such that $z_{q} \phi=v_{j}$ for some $j$. (Such a $q$ exists since $z_{p} \phi=v_{u-i}$.) Then

$$
z_{0} \phi \rightarrow \cdots \rightarrow z_{q} \phi=v_{j} \rightarrow z_{q+1} \phi=v_{j-1} \rightarrow \cdots \rightarrow z_{p} \phi=v_{j-(p-q)}=v_{u-i} .
$$

Then, for the branch $\left(\ldots w_{q}=z_{0} \phi \ldots w_{0}=z_{q} \phi=v_{j} \ldots v_{0}=y_{t}\right]$ of $\theta, \phi$ maps $\left[z_{0} \ldots z_{q}\right]$ onto $\left[w_{q} \ldots w_{0}=v_{j}\right]$, $\phi$ maps $\left[z_{q} \ldots z_{p}=x_{i}\right]$ onto $\left[v_{j} \ldots v_{u-i}\right]$ (if $q<p$ ), and $j-p+q=u-i$ (since $\left.v_{j-(p-q)}=v_{u-i}\right)$.

Thus (3a) or (3b) holds, and so exactly one of them holds since (3a) and (3b) are mutually exclusive.

Conversely, suppose (1)-(3) are satisfied. Then $\phi$ is a homomorphism from $D(\gamma)$ to $D(\delta)$ by an argument similar to the one used in the proof of Lemma 3.6.

Figure 3.8 illustrates how the maximal right ray $\eta=\left[x_{0} x_{1} x_{2} \ldots\right\rangle$ from Lemma 3.11 is mapped by a homomorphism.

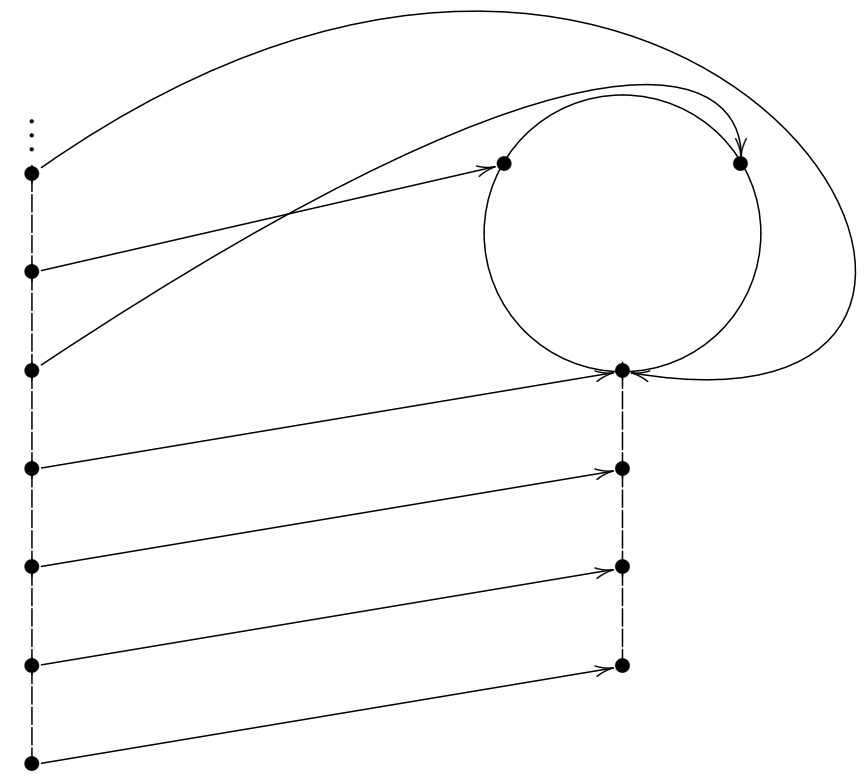

Figure 3.8: A homomorphism acting on a maximal right ray in Lemma 3.11.

Lemma 3.12. Let $\gamma$ and $\delta$ be connected components of $\alpha \in T(X)$ such that $\gamma$ is of type rro with a maximal right ray $\eta=\left[x_{0} x_{1} x_{2} \ldots\right\rangle$, and $\delta$ has a double ray $\pi=\left\langle\ldots y_{-1} y_{0} y_{1} \ldots\right\rangle$. Let $\phi: \operatorname{dom}(\gamma) \rightarrow \operatorname{dom}(\delta)$ such that $\phi$ maps $\eta$ onto $\left[y_{t} y_{t+1} \ldots\right\rangle$ for some $t$. Then $\phi$ is a homomorphism from $D(\gamma)$ to $D(\delta)$ if and only if for every branch $\tau=\left[z_{0} \ldots z_{p}=x_{i}\right]$ of $\eta$, exactly one of the following holds:

(a) $\phi$ maps $\tau$ onto $\left[y_{t+i-p} \ldots y_{t+i}\right]$; or

(b) There is a branch $\mu=\left(\ldots w_{q} \ldots w_{0}=y_{t+i-p+q}\right]$ of $\pi$ with $1 \leq q \leq p$ such that $\phi$ maps $\left[z_{0} \ldots z_{q}\right]$ onto $\left[w_{q} \ldots w_{0}=y_{t+i-p+q}\right]$ and if $q<p$ then $\phi$ maps $\left[z_{q} \ldots z_{p}=x_{i}\right]$ onto $\left[y_{t+i-p+q} \ldots y_{t+i}\right]$. 
Proof. To prove $(\Rightarrow)$, follow the proof of Lemma 3.8(1). The converse follows easily from the fact that $\gamma$ is the join of $\eta$ and its (necessarily finite) branches (see Proposition 2.13).

Lemma 3.13. Let $\gamma$ and $\delta$ be connected components of $\alpha \in T(X)$ such that $\gamma$ is of type rro and has a right ray $\eta=\left[x_{0} x_{1} x_{2} \ldots\right\rangle$, and $\delta$ has a double ray $\pi=\left\langle\ldots y_{-1} y_{0} y_{1} \ldots\right\rangle$ such that for some $t$ and $u \geq 1, \phi$ maps $\left[x_{u} x_{u+1} \ldots\right\rangle$ onto $\left[y_{t} y_{t+1} \ldots\right\rangle, x_{u-1} \notin \operatorname{dom}(\pi)$, and $x_{0} \phi$ does not lie on an infinite branch of $\pi$. Then $\phi$ is a homomorphism from $D(\gamma)$ to $D(\delta)$ if and only if the following conditions are satisfied:

(1) There is a finite branch $\kappa=\left[\ldots v_{0} \ldots v_{u}=y_{t}\right]$ of $\pi$ such that $\phi$ maps $\left[x_{0} \ldots x_{u}\right]$ onto $\left[v_{0} \ldots v_{u}\right]$

(2) If $\tau=\left[z_{0} \ldots z_{p}=x_{i}\right]$ is a branch of $\eta$ with $i \geq u$, then exactly one of the following holds:

(a) $\phi$ maps $\tau$ onto $\left[y_{t+i-u-p} \ldots y_{t+i-u}\right]$; or

(b) There is a branch $\left(\ldots w_{q} \ldots w_{0}=y_{t+i-u-p+q}\right]$ of $\pi$ with $1 \leq q \leq p$ such that $\phi$ maps $\left[z_{0} \ldots z_{q}\right]$ onto $\left[w_{q} \ldots w_{0}=y_{t+i-u-p+q}\right]$ and if $q<p$ then $\phi$ maps $\left[z_{q} \ldots z_{p}=x_{i}\right]$ onto $\left[y_{t+i-u-p+q} \ldots y_{t+i-u}\right]$;

(3) If $\tau=\left[z_{0} \ldots z_{p}=x_{i}\right]$ is a branch of $\eta$ with $i<u$, then exactly one of the following holds:

(a) $\phi$ maps $\tau$ onto $\left[v_{i-p} \ldots v_{i}\right]$; or

(b) There is a branch $\left(\ldots w_{q} \ldots w_{1} w_{0}=v_{i-p+q} \ldots v_{u}=y_{t}\right]$ of $\pi$ with $1 \leq q \leq p$ and $w_{1} \notin \operatorname{dom}(\kappa)$ such that $\phi$ maps $\left[z_{0} \ldots z_{q}\right]$ onto $\left[w_{q} \ldots w_{0}=v_{i-p+q}\right]$ and if $q<p$ then $\phi \operatorname{maps}\left[z_{q} \ldots z_{p}=x_{i}\right]$ onto $\left[v_{i-p+q} \ldots v_{i}\right]$.

Proof. Suppose $\phi$ is a homomorphism from $D(\gamma)$ to $D(\delta)$. Then (1) is satisfied since $x_{0} \phi$ lies on some finite branch of $\pi$ (by Proposition 2.13 and the assumption about $x_{0}$ ).

Let $\tau=\left[z_{0} \ldots z_{p}=x_{i}\right]$ be a branch of $\eta$ with $i \geq u$. Since $\phi$ preserves edges, we have

$$
z_{0} \phi \rightarrow z_{1} \phi \rightarrow \cdots \rightarrow z_{p} \phi=x_{i} \phi=y_{t+i-u}
$$

where the last equality is true since $x_{u} \phi=y_{t}$ and $\phi$ maps $\left[x_{u} x_{u+1} \ldots\right\rangle$ onto $\left[y_{t} y_{t+1} \ldots\right\rangle$. Suppose $z_{0} \phi$ lies on $\pi$. Then, by (3.8), $z_{0} \phi=y_{t+i-u-p}$ and $\phi$ maps $\tau$ onto $\left[y_{t+i-u-p} \ldots y_{t+i-u}\right]$.

Suppose $z_{0} \phi$ does not lie on $\pi$. Then, by Proposition 2.13, $z_{0} \phi$ lies on some branch $\mu=$ $\left(\ldots w_{2} w_{1} w_{0}=y_{j}\right]$ of $\pi$. Let $z_{0} \phi=w_{q}$. Then $q \geq 1$ (since $z_{0} \phi$ does not lie on $\pi$ ) and

$$
z_{0} \phi=w_{q} \rightarrow \cdots \rightarrow z_{q} \phi=w_{0}=y_{j} \rightarrow z_{q+1} \phi=y_{j+1} \rightarrow \cdots \rightarrow z_{p} \phi=y_{j+p-q} .
$$

Since $y_{j+p-q}=z_{p} \phi=x_{i} \phi=y_{t+i-u}$, we have $j=t+i-u-p+q$. Thus, by (3.9), $\phi$ maps $\left[z_{0} \ldots z_{q}\right]$ onto $\left[w_{q} \ldots w_{0}=y_{t+i-u-p+q}\right]$, and if $q<p$ then $\phi$ maps $\left[z_{q} \ldots z_{p}=x_{i}\right]$ onto $\left[y_{t+i-u-p+q} \ldots y_{t+i-u}\right]$. Hence (2a) or (2b) holds. Thus exactly one of them holds since (2a) and (2b) are mutually exclusive. We have proved (2). The proof of (3) is similar.

Conversely, suppose (1)-(3) are satisfied. Then $\phi$ is a homomorphism from $D(\gamma)$ to $D(\delta)$ by an argument similar to the one used in the proof of Lemma 3.6.

Figure 3.9 illustrates how the maximal right ray $\eta=\left[x_{0} x_{1} x_{2} \ldots\right\rangle$ from Lemma 3.13 and its branch are mapped by a homomorphism, where we assume that $\eta$ is the vertical maximal right ray in the figure and $i \geq u$ (see (2) of Lemma 3.13). 


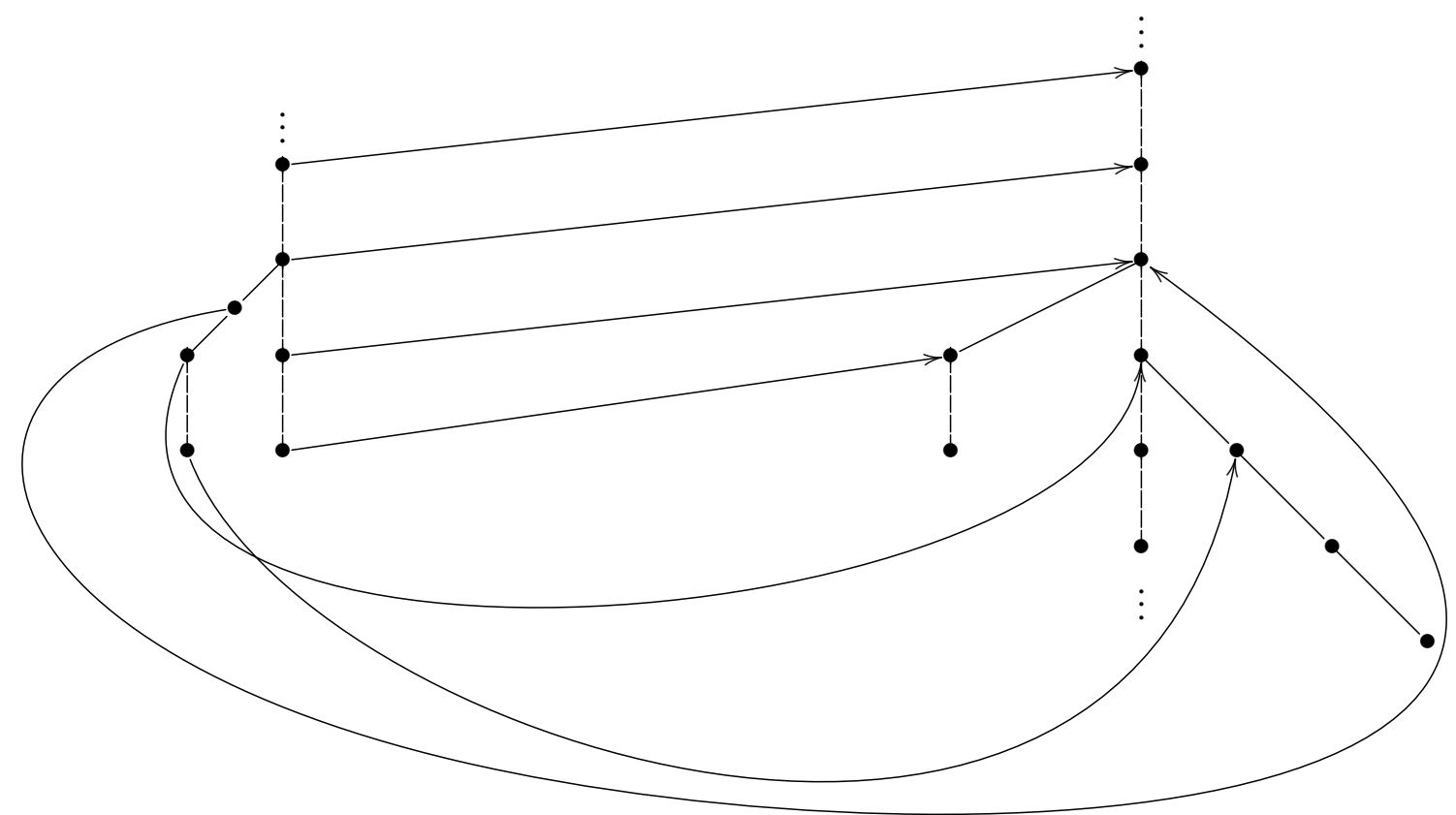

Figure 3.9: A homomorphism acting on a maximal right ray and its branch in Lemma 3.13.

Lemma 3.14. Let $\gamma$ and $\delta$ be connected components of $\alpha \in T(X)$ of type rro such that $\gamma$ has a maximal right ray $\eta=\left[x_{0} x_{1} x_{2} \ldots\right]$ and $\delta$ has a maximal right ray $\xi=\left[y_{0} y_{1} y_{2} \ldots\right\rangle$. Let $\phi: \operatorname{dom}(\gamma) \rightarrow \operatorname{dom}(\delta)$ such that $\phi$ maps $\eta$ onto $\left[y_{t} y_{t+1} \ldots\right\rangle$ for some $t \geq 0$. Then $\phi$ is a homomorphism from $D(\gamma)$ to $D(\delta)$ if and only if for every branch $\tau=\left[z_{0} \ldots z_{p}=x_{i}\right]$ of $\eta$, exactly one of the following holds:

(a) $\phi$ maps $\tau$ onto $\left[y_{t+i-p} \ldots y_{t+i}\right]$; or

(b) There is a branch $\kappa=\left[\ldots w_{0} \ldots w_{q}=y_{t+i-p+q}\right]$ of $\xi$ with $1 \leq q \leq p$ such that $\phi$ maps $\left[z_{0} \ldots z_{q}\right]$ onto $\left[w_{0} \ldots w_{q}=y_{t+i-p+q}\right]$ and if $q<p$ then $\phi$ maps $\left[z_{q} \ldots z_{p}=x_{i}\right]$ onto $\left[y_{t+i-p+q} \ldots y_{t+i}\right]$.

Proof. Suppose $\phi$ is a homomorphism from $D(\gamma)$ to $D(\delta)$. Let $\tau=\left[z_{0} \ldots z_{p}=x_{i}\right]$ be a branch of $\eta$. Since $\phi$ preserves edges, we have

$$
z_{0} \phi \rightarrow z_{1} \phi \rightarrow \cdots \rightarrow z_{p} \phi=x_{i} \phi=y_{t+i},
$$

where the last equality is true since $x_{0} \phi=y_{t}$ and $\phi$ maps $\eta$ onto $\left[y_{t} y_{t+1} \ldots\right\rangle$. Suppose $z_{0} \phi$ lies on $\xi$. Then, by (3.10), $z_{0} \phi=y_{t+i-p}$ and $\phi$ maps $\tau$ onto $\left[y_{t+i-p} \ldots y_{t+i}\right]$.

Suppose $z_{0} \phi$ does not lie on $\xi$. Then there exists a branch $\kappa=\left[\ldots w_{0} \ldots w_{q}=y_{j}\right]$ of $\xi$ (see by Proposition 2.13) such that $q \geq 1$ and $z_{0} \phi=w_{0}$. Hence

$$
z_{0} \phi=w_{0} \rightarrow \cdots \rightarrow z_{q} \phi=w_{q}=y_{j} \rightarrow z_{q+1} \phi=y_{j+1} \rightarrow \cdots \rightarrow z_{p} \phi=y_{j+p-q} .
$$

Since $y_{j+p-q}=z_{p} \phi=x_{i} \phi=y_{t+i}$, we have $j=t+i-p+q$. Thus, by (3.11), $\phi$ maps $\left[z_{0} \ldots z_{q}\right]$ onto $\left[w_{0} \ldots w_{q}=y_{t+i-p+q}\right]$, and if $q<p$ then $\phi$ maps $\left[z_{q} \ldots z_{p}=x_{i}\right]$ onto $\left[y_{t+i-p+q} \ldots y_{t+i}\right]$. Hence (a) or (b) holds. Thus exactly one of them holds since (a) and (b) are mutually exclusive. 
The converse follows easily from the fact that $\gamma$ is the join of $\eta$ and its (necessarily finite) branches (see Proposition 2.13).

Figure 3.10 illustrates how the maximal right ray $\eta=\left[x_{0} x_{1} x_{2} \ldots\right\rangle$ from Lemma 3.14 and its branch are mapped by a homomorphism, where we assume that $\eta$ and $\delta$ are the vertical maximal right rays in the figure.

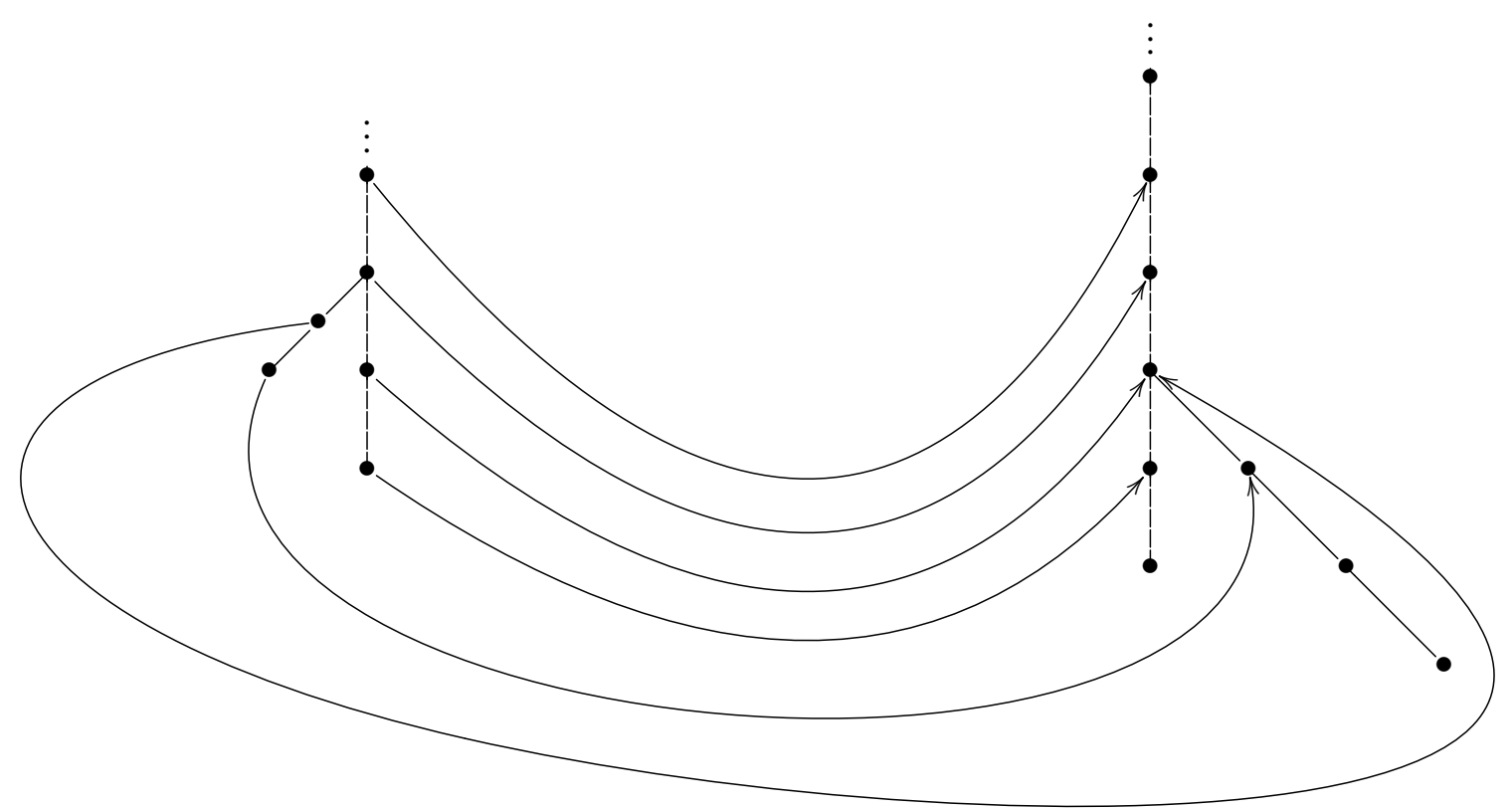

Figure 3.10: A homomorphism acting on a maximal right ray and its branch in Lemma 3.14.

\section{The Characterization Theorem}

The description of the elements $\beta \in C(\alpha)$, where $\alpha \in T(X)$, reduces to the description of the graph homomorphisms from $D(\gamma)$ to $D(\delta)$, where $\gamma$ and $\delta$ are connected components of $\alpha$. This follows from Proposition 4.1 below.

Proposition 4.1. Let $\alpha, \beta \in T(X)$. Then $\beta \in C(\alpha)$ if and only if for every connected component $\gamma$ of $\alpha$, there exists a connected component $\delta$ of $\alpha$ such that $\left.\beta\right|_{\operatorname{dom}(\gamma)}$ is a graph homomorphism from $D(\gamma)$ to $D(\delta)$.

Proof. Suppose $\beta \in C(\alpha)$. Then, by Proposition 3.1, $\beta$ is a homomorphism from $D(\alpha)$ to $D(\alpha)$. Let $\gamma$ be a connected component of $\alpha$ and let $x \in \operatorname{dom}(\gamma)$. Then, by Proposition 2.5, $x \beta \in \operatorname{dom}(\delta)$ for some connected component $\delta$ of $\alpha$. We claim that $(\operatorname{dom}(\gamma)) \beta \subseteq \operatorname{dom}(\delta)$. Let $z \in \operatorname{dom}(\gamma)$. Since $\gamma$ is connected, $x \alpha^{k}=x \gamma^{k}=z \gamma^{m}=z \alpha^{m}$ for some integers $k, m \geq 0$. Since $\beta \in C(\alpha)$, we have $\alpha \beta=\beta \alpha$, and so $(z \beta) \alpha^{m}=\left(z \alpha^{m}\right) \beta=\left(x \alpha^{k}\right) \beta=(x \beta) \alpha^{k}$, which implies that $z \beta$ and $x \beta$ are in the domain of the same connected component of $\alpha$, that is, $z \beta \in \operatorname{dom}(\delta)$. The claim has been proved. Then $\left.\beta\right|_{\operatorname{dom}(\gamma)}$ is a homomorphism from $D(\gamma)$ to $D(\delta)$ by the claim and the fact that $\beta$ is a homomorphism from $D(\alpha)$ to $D(\alpha)$. 
Conversely, suppose that the given condition is satisfied. Suppose $y \stackrel{\alpha}{\rightarrow} z$. Then $y, z \in \operatorname{dom}(\gamma)$ for some connected component $\gamma$ of $\alpha$. It is given that $\phi=\left.\beta\right|_{\operatorname{dom}(\gamma)}$ is a homomorphism from $D(\gamma)$ to $D(\delta)$ for some connected component $\delta$ of $\alpha$. Thus $y \beta=y \phi \stackrel{\delta}{\rightarrow} z \phi=z \beta$, implying $y \beta \stackrel{\alpha}{\rightarrow} z \beta$. Hence $\beta$ is a homomorphism from $D(\alpha)$ to $D(\alpha)$, and so $\beta \in C(\alpha)$ by Proposition 3.1.

In view of Proposition 4.1, we can now characterize the elements of $C(\alpha)$ using the results of Section 3.

Theorem 4.2. Let $\alpha, \beta \in T(X)$, where $X$ is an arbitrary set. Then $\beta \in C(\alpha)$ if and only if for every connected component $\gamma$ of $\alpha$, there exists a connected component $\delta$ of $\alpha$ such that the following conditions are satisfied for $\phi=\left.\beta\right|_{\operatorname{dom}(\gamma)}$ :

(1) $\operatorname{im}(\phi) \subseteq \operatorname{dom}(\delta)$;

(2) If $\gamma$ has a cycle $\sigma=\left(x_{0} \ldots x_{k-1}\right)$, then $\delta$ has a cycle $\theta=\left(y_{0} \ldots y_{m-1}\right)$ and (1)-(4) of Lemma 3.4 are satisfied;

(3) If $\gamma$ has a double ray $\omega=\left\langle\ldots x_{-1} x_{0} x_{1} \ldots\right\rangle$, then exactly one of the following holds:

(a) $\delta$ has a cycle $\theta=\left(y_{0} \ldots y_{m-1}\right)$, $\phi$ double-wraps $\omega$ around $\theta$ at some $y_{t}$, and (1) and (2) of Lemma 3.6 are satisfied;

(b) $\delta$ has a cycle $\theta=\left(y_{0} \ldots y_{m-1}\right)$, there exists $u$ such that $\phi$ wraps $\left[x_{u} x_{u+1} \ldots\right\rangle$ around $\theta$ at some $y_{t}$ and $x_{u-1} \notin \operatorname{dom}(\theta)$, and (1)-(5) of Lemma 3.7 are satisfied; or

(c) $\delta$ has a double ray $\pi=\left\langle\ldots y_{-1} y_{0} y_{1} \ldots\right\rangle$ such that $\phi$ maps $\omega$ onto $\pi$ at some $y_{t}$ and (1) and (2) of Lemma 3.8 are satisfied;

(4) If $\gamma$ is of type rro and has a maximal right ray $\eta=\left[x_{0} x_{1} x_{2} \ldots\right\rangle$, then exactly one of the following holds:

(a) $\delta$ has a cycle $\theta=\left(y_{0} \ldots y_{m-1}\right)$, $\phi$ wraps $\eta$ around $\theta$ at some $y_{t}$, and for every branch $\tau=\left[z_{0} \ldots z_{p}=x_{i}\right]$ of $\eta$, (a) or (b) of Lemma 3.10 holds;

(b) $\delta$ has a cycle $\theta=\left(y_{0} \ldots y_{m-1}\right)$, there exists $u \geq 1$ such that $\phi$ wraps $\left[x_{u} x_{u+1} \ldots\right\rangle$ around $\theta$ at some $y_{t}$ and $x_{u-1} \notin \operatorname{dom}(\theta)$, and (1)-(3) of Lemma 3.11 are satisfied;

(c) $\delta$ has a double ray $\pi=\left\langle\ldots y_{-1} y_{0} y_{1} \ldots\right\rangle$ such that $\phi$ maps $\eta$ onto $\left[y_{t} y_{t+1} \ldots\right\rangle$ for some $t$ and for every branch $\tau=\left[z_{0} \ldots z_{p}=x_{i}\right]$ of $\eta$, (a) or (b) of Lemma 3.12 holds;

(d) $\delta$ has a double ray $\pi=\left\langle\ldots y_{-1} y_{0} y_{1} \ldots\right\rangle$ such that for some $t$ and $u \geq 1, \phi$ maps $\left[x_{u} x_{u+1} \ldots\right\rangle$ onto $\left[y_{t} y_{t+1} \ldots\right\rangle, x_{u-1} \notin \operatorname{dom}(\pi), x_{0}$ does not lie on an infinite branch of $\pi$, and (1)-(3) of Lemma 3.13 are satisfied; or

(e) $\delta$ is of type rro and has a maximal right ray $\xi=\left[y_{0} y_{1} y_{2} \ldots\right\rangle$ such that $\phi$ maps $\eta$ onto $\left[y_{t} y_{t+1} \ldots\right\rangle$ for some $t \geq 0$ and for every branch $\tau=\left[z_{0} \ldots z_{p}=x_{i}\right]$ of $\eta$, (a) or (b) of Lemma 3.14 holds.

Proof. Suppose $\beta \in C(\alpha)$. Let $\gamma$ be a connected component of $\alpha$ and let $\phi=\left.\beta\right|_{\operatorname{dom}(\gamma)}$. Then, by Proposition 4.1, there is a connected component $\delta$ of $\alpha$ such that $\phi$ is a homomorphism from $D(\gamma)$ to $D(\delta)$, and so (1) is satisfied. Further, (2) is satisfied by Lemma 3.4; (3) by Lemmas 3.6-3.8; and (4) by Lemma 3.10-3.14.

Conversely, suppose that for every connected component $\gamma$ of $\alpha$, there exists a connected component $\delta$ of $\alpha$ such that (1)-(4) are satisfied for $\phi=\left.\beta\right|_{\operatorname{dom}(\gamma)}$. Let $\gamma$ be a connected component of 
$\alpha$. Then there is a connected component $\delta$ of $\alpha$ such that (1)-(4) are satisfied for $\phi=\left.\beta\right|_{\operatorname{dom}(\gamma)}$. Then $\phi: \operatorname{dom}(\gamma) \rightarrow \operatorname{dom}(\delta)$ by (1). We want to prove that $\phi$ is a homomorphism from $D(\gamma)$ to $D(\delta)$. If $\gamma$ has a cycle, then $\phi$ is a homomorphism by (2) and Lemma 3.4. If $\gamma$ has a double ray, then $\phi$ is a homomorphism by (3) and Lemmas 3.5-3.8. Suppose $\gamma$ does not have a cycle or a double ray. Then $\gamma$ is of type rro by Proposition 2.10 and Definition 2.11. Thus $\phi$ is a homomorphism by (3) and Lemmas 3.9-3.14. Hence $\beta \in C(\alpha)$ by Proposition 4.1.

\section{Special Cases}

Theorem 4.2 can be applied to the case when $X$ is finite and to particular types of transformations in $T(X)$. In this section, we provide some examples of these applications.

\section{The finite case.}

Suppose $X$ is finite and let $\alpha \in T(X)$. Then $\alpha$ cannot have any rays, and so every connected component of $\alpha \in T(X)$ is the join of its unique cycle and the finite branches of the cycle. This gives the following corollary to Theorem 4.2 (see [31, Theorem 2]).

Corollary 5.1. Let $\alpha, \beta \in T(X)$, where $X$ is a finite set. Then $\beta \in C(\alpha)$ if and only if for every connected component $\gamma$ of $\alpha$ with cycle $\sigma=\left(x_{0} \ldots x_{k-1}\right)$, there exists a connected component $\delta$ of $\alpha$ with cycle $\theta=\left(y_{0} \ldots y_{m-1}\right)$ such that the following conditions are satisfied for $\phi=\left.\beta\right|_{\operatorname{dom}(\gamma)}$ :

(1) $\operatorname{im}(\phi) \subseteq \operatorname{dom}(\delta)$;

(2) $m$ divides $k$;

(3) $\phi$ wraps $\sigma$ around $\theta$ at some $y_{t}$;

(4) If $\tau=\left[z_{0} \ldots z_{p}=x_{i}\right]$ is a finite branch of $\sigma$, then exactly one of the following holds:

(a) $\phi$ wraps $\tau$ around $\theta$ at $y_{t+i-p}$; or

(b) There is a finite branch $\mu=\left[\ldots w_{q} \ldots w_{0}=y_{j}\right]$ of $\theta$ with $1 \leq q \leq p$ and $j+p-q \equiv$ $t+i(\bmod m)$ such that $\phi$ maps $\left[z_{0} \ldots z_{q}\right]$ onto $\left[w_{q} \ldots w_{0}=y_{j}\right]$ and if $q<p$ then $\phi$ wraps $\left[z_{q} \ldots z_{p}=x_{i}\right]$ around $\theta$ at $y_{j}$.

\section{Idempotents.}

Let $\varepsilon \in T(X)$ (where $X$ is arbitrary) be an idempotent. Then for every $x \in X$ and $y=x \varepsilon$, $y \varepsilon=(x \varepsilon) \varepsilon)=x(\varepsilon \varepsilon)=x \varepsilon=y$. It easily follows that if $\gamma$ is a connected component of $\varepsilon$, then $\operatorname{im}(\gamma)=\{y\}$, for some $y \in X$, and $\gamma$ is the joint of the 1-cycle $(y)$ and some (possibly none) finite branches $[x y]$ of length 2 (see Figure 5.1).

Let $\gamma$ and $\delta$ be connected components of an idempotent $\varepsilon$, with cycles $(y)$ and $(z)$, respectively. If $[x y]$ is a finite branch of $(y)$, then $\beta \in T(X)$ satisfies (3b) of Lemma 3.4 if and only if $y \beta=z$ and $x \beta \in \operatorname{dom}(\delta)$. Therefore, Theorem 4.2 applied to an idempotent $\varepsilon$ gives the following corollary (see [5, Lemma 2.2] and [24, Theorem 2.1]).

Corollary 5.2. Let $\varepsilon, \beta \in T(X)$, where $\varepsilon$ is an idempotent. Then $\beta \in C(\varepsilon)$ if and only if for every connected component $\gamma$ of $\varepsilon$ with cycle $(y)$, there exists a connected component $\delta$ of $\varepsilon$ with cycle $(z)$ such that $y \beta=z$ and $(\operatorname{dom}(\gamma)) \beta \subseteq \operatorname{dom}(\delta)$. 


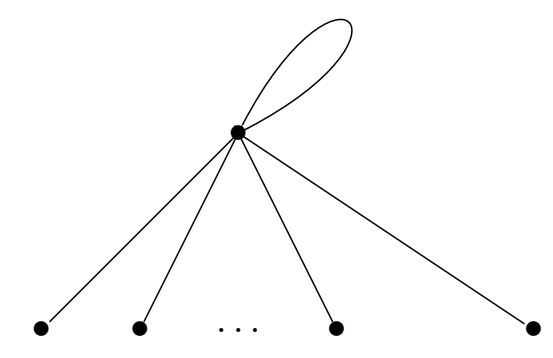

Figure 5.1: A connected component of an idempotent.

\section{Injective transformations.}

Any connected component of an injective transformations $\alpha \in T(X)$ is a cycle, a double ray, or a right ray (see Figure 5.2).

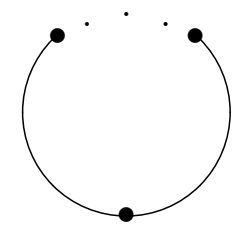

Figure 5.2: Connected components of an injective transformation.

Applying Theorem 4.2 to an injective transformation, we obtain the following corollary. (See [26, Theorem 3.9] where the centralizer of an injective $\alpha$ is described relative to the semigroup of injective transformations on $X$.)

Corollary 5.3. Let $\alpha, \beta \in T(X)$ such that $\alpha$ is injective. Then $\beta \in C(\alpha)$ if and only if conditions are satisfied:

(1) For every cycle $\sigma=\left(x_{0} \ldots x_{k-1}\right)$ in $\alpha$, there is a cycle $\theta=\left(y_{0} \ldots y_{m-1}\right)$ in $\alpha$ such that $m$ divides $k$ and $\beta$ wraps $\sigma$ around $\theta$ at some $y_{t}$;

(2) For every a double ray $\omega=\left\langle\ldots x_{-1} x_{0} x_{1} \ldots\right\rangle$ in $\alpha$, exactly one of the following holds:

(a) There is a cycle $\theta=\left(y_{0} \ldots y_{m-1}\right)$ in $\alpha$ such that $\beta$ double-wraps $\omega$ around $\theta$ at some $y_{t}$;

(b) There is a double ray $\pi=\left\langle\ldots y_{-1} y_{0} y_{1} \ldots\right\rangle$ in $\alpha$ such that $\beta$ maps $\omega$ onto $\pi$ at some $y_{t}$;

(3) For every maximal right ray $\eta=\left[x_{0} x_{1} x_{2} \ldots\right]$ in $\alpha$, exactly one of the following holds:

(a) There is a cycle $\theta=\left(y_{0} \ldots y_{m-1}\right)$ in $\alpha$ such that $\beta$ wraps $\eta$ around $\theta$ at some $y_{t}$;

(b) There is a double ray $\pi=\left\langle\ldots y_{-1} y_{0} y_{1} \ldots\right\rangle$ in $\alpha$ such that $\beta$ maps $\eta$ onto $\left[y_{t} y_{t+1} \ldots\right\rangle$ for some t;

(c) There is a maximal right ray $\xi=\left[y_{0} y_{1} y_{2} \ldots\right\rangle$ in $\alpha$ such that $\beta$ maps $\eta$ onto $\left[y_{t} y_{t+1} \ldots\right\rangle$ for some $t \geq 0$. 


\section{Permutations.}

Since any connected component of a permutation $\alpha$ on $X$ is a cycle, Corollary 5.3 implies the following result.

Corollary 5.4. Let $\alpha, \beta \in T(X)$ such that $\alpha$ is a permutation. Then $\beta \in C(\alpha)$ if and only if for every cycle $\sigma=\left(x_{0} \ldots x_{k-1}\right)$ in $\alpha$, there is a cycle $\theta=\left(y_{0} \ldots y_{m-1}\right)$ in $\alpha$ such that $m$ divides $k$ and $\beta$ wraps $\sigma$ around $\theta$ at some $y_{t}$.

\section{Problems}

In the previous sections, we described the centralizer of an arbitrary $\alpha \in T(X)$. Of course, this is just the first step towards a description of the structure (from a semigroup theoretical point of view) of $C(\alpha)$. Given the complexity of Theorem 4.2, however, such a description in all generality does not appear to be feasible. What can be done, though, is to provide such descriptions for particular types of transformations. For various particular types, it should be possible to obtain results similar to those contained in [4] and [5], and hence provide generalizations to $C(\alpha)$ of many results originally proved for the special case of $T(X)=C\left(\operatorname{id}_{X}\right)$. More specifically, given a transformation $\alpha \in T(X)$ of a certain pre-defined type, we would like to see the following program fulfilled:

1. Describe the automorphisms of $C(\alpha)$. (This has been done for the idempotents [4]; the most natural candidates to consider next are the injective transformations [26].)

2. Describe Green's relations in $C(\alpha)$.

3. Let $T$ be one of Green's relations. Characterize the transformations $\alpha \in T(X)$ (of the given type) such that if $\beta, \gamma \in C(\alpha)$ and $\beta, \gamma$ are $T$-related in $T(X)$, then $\beta, \gamma$ are $T$-related in $C(\alpha)$.

4. Characterize the transformations $\alpha \in T(X)$ (of the given type) such that $\mathcal{D}=\mathcal{J}$ in $C(\alpha)$.

5. Characterize the transformations $\alpha \in T(X)$ (of the given type) such that the partial order of $J$-classes in $C(\alpha)$ is a chain.

6. For $\alpha \in T(X)$ (of the given type), describe the regular elements in $C(\alpha)$, and characterize those $\alpha$ for which $C(\alpha)$ is regular.

7. For $\alpha \in T(X)$ (of the given type), describe the semigroup generated by the idempotents of $C(\alpha)$.

8. Repeat problems 1-7 for $C_{S}(\alpha)$, where $\alpha \in T(X)$ is a transformation of a given type, $S$ is a subsemigroup of $T(X)$, and $C_{S}(\alpha)=\{\beta \in S: \alpha \beta=\beta \alpha\}$ is the centralizer of $\alpha$ relative to $S$.

The program outlined above has been carried out for the idempotents and, in part, for the injective transformations [26]. We introduce some other types of transformations that appear to be especially interesting and promising.

\section{Howie's transformations.}


Let $X$ be an infinite set. For $\alpha \in T(X)$ define the following sets:

$$
S(\alpha)=\{x \in X: x \alpha \neq x\}, \quad Z(\alpha)=X \backslash \operatorname{im}(\alpha), \quad C l(\alpha)=\bigcup\left\{t \alpha^{-1}:\left|t \alpha^{-1}\right| \geq 2\right\} .
$$

By Howie's celebrated result [15], we know that the semigroup generated by the idempotents of $T(X)$ consists of the identity of $T(X)$ together with the elements of the following sets:

$$
\begin{aligned}
& F(X)=\left\{\alpha \in T(X):|S(\alpha)|<\aleph_{0} \text { and }|Z(\alpha)|>0\right\} \\
& Q(X)=\left\{\alpha \in T(X):|S(\alpha)|=|Z(\alpha)|=|C l(\alpha)| \geq \aleph_{0}\right\}
\end{aligned}
$$

Problem. Carry out (part of) the program outlined at the beginning of this section for the transformations in $F(X)[Q(X)]$, where $X$ is infinite $[X=\mathbb{N}]$.

\section{Transformations with stabilizers.}

Following [40], we define the stabilizer of $\alpha \in T(X)$ as the smallest integer $s \geq 0$ such that $\operatorname{im}\left(\alpha^{s}\right)=\operatorname{im}\left(\alpha^{s+1}\right)$. If such an $s$ does not exist, we say that $\alpha$ has no stabilizer. The transformations that have the stabilizer have been described in terms of their digraphs in [8].

Problem. Carry out (part of) the program outlined at the beginning of this section for the transformations with stabilizer, for an infinite $X[X=\mathbb{N}]$.

\section{Cayley functions.}

A transformation $\alpha \in T(X)$ is called a Cayley function if there is a binary operation $*$ on $X$ such that $(X, *)$ is a semigroup and $\alpha$ is an inner translation of the semigroup; that is, there exists $a \in X$ such that for every $x \in X, x \alpha=x * a$. The algebraic description of the Cayley functions has been given in [40]. The digraphs of the Cayley functions have been characterized in [8].

Problem. Carry out (part of) the program outlined at the beginning of this section for the Cayley functions [Cayley functions with stabilizer], for an infinite $X[X=\mathbb{N}]$.

\section{Transformations with large contraction index and collapse.}

Let $X$ be an infinite set and let $\alpha \in T(X)$. The kernel of $\alpha$ is the relation $\{(x, y) \in X \times X$ : $x \alpha=y \alpha\}$. By $X / \operatorname{ker}(\alpha)$ we denote the partition of $X$ induced by $\operatorname{ker}(\alpha)$, that is, $X / \operatorname{ker}(\alpha)=$ $\left\{x \alpha^{-1}: x \in X\right\}$. The defect $d(\alpha)$ of $\alpha$ is the cardinal $|X \backslash \operatorname{im}(\alpha)|$; the contraction index $k(\alpha)$ of $\alpha$ is the number of classes in $X / \operatorname{ker}(\alpha)$ of size $|X|$; and the collapse $c(\alpha)$ of $\alpha$ is the cardinal $\left|X \backslash T_{\alpha}\right|$, where $T_{\alpha}$ is a cross-section of the partition $X / \operatorname{ker}(\alpha)$.

The importance of these parameters comes from the following two results proved in [17] (see also [9]), where it has been established when the symmetric group $\operatorname{Sym}(X)$ [the set of idempotents $E(X)$ in $T(X)$ ], with two extra elements, generates $T(X)$.

Theorem 6.1. ([17, Theorem 4.1]) Let $X$ be an infinite set such that $|X|$ is a regular cardinal. Then, for all $\mu, \nu \in T(X),\langle\operatorname{Sym}(X), \mu, \nu\rangle=T(X)$ if and only if $\{\mu, \nu\}$ consists of an injection of defect $|X|$ and a surjection of contraction index $|X|$.

Theorem 6.2. ([17, Theorem 6.1]) Let $X$ be an infinite set and let $E(X)$ denote the set of idempotents of $T(X)$. Then, for all $\mu, \nu \in T(X),\langle E(X), \mu, \nu\rangle=T(X)$ if and only if $\{\mu, \nu\}$ consists of an injection of defect $|X|$ and a surjection of collapse $|X|$.

Problem. Carry out (part of) the program outlined at the beginning of this section for the surjective transformations $\alpha \in T(X)$ such that every class in $X / \operatorname{ker}(\alpha)$ has size $|X|[\alpha$ has collapse $|X|]$. 


\section{Transformations associated with maximal subsemigroups of $T(X)$}

We say that $S \leq T(X)$ is a maximal subsemigroup of $T(X)$ if $S \neq T(X)$ and for all $\alpha \in T(X) \backslash S, S$ together with $\alpha$ generate $T(X)$. For $\alpha \in T(X)$, let $d(\alpha), c(\alpha)$, and $k(\alpha)$ be, respectively, the defect, collapse, and contraction index of $\alpha$ (as defined above). For an infinite set $X$ of regular cardinality, all maximal subsemigroups of $T(X)$ containing $\operatorname{Sym}(X)$ have been described in [12]:

Theorem 6.3. Let $X$ be any infinite set such that $|X|$ is a regular cardinal, and let $M$ be a subsemigroup of $T(X)$ containing the symmetric group $\operatorname{Sym}(X)$. Then $M$ is maximal if and only if $M$ is one of the following semigroups:

(1) $\{\alpha \in T(X): c(\alpha)<\mu$ or $d(\alpha) \geq \mu\}$, for some infinite cardinal $\mu \leq|X|$;

(2) $\{\alpha \in T(X): c(\alpha)=0$ ord $(\alpha)>0\}$;

(3) $\{\alpha \in T(X): k(\alpha)<|X|\}$;

(4) $\{\alpha \in T(X): c(\alpha) \geq \mu$ or $d(\alpha)<\mu\}$, for some infinite cardinal $\mu \leq|X|$;

(5) $\{\alpha \in T(X): c(\alpha)>0$ ord $(\alpha)=0\}$.

Problem. Carry out (part of) the program outlined at the beginning of this section for the transformations in each one of the sets defined in Theorem 6.3.

\section{Endomorphisms.}

For a mathematical structure $\mathcal{A}$ with universe $X$, let $\operatorname{End}(\mathcal{A})$ denote the monoid of endomorphisms of $\mathcal{A}$ (see [6] and [7]). Then $\operatorname{End}(\mathcal{A})$ is a subsemigroup of $T(X)$. For example, if $\mathcal{A}=(X, \rho, R)$, where $\mathrm{X}$ is a set, $\rho$ is an equivalence relation on $X$, and $R$ is a cross-section of $X / \rho$, then the elements of $\operatorname{End}(\mathcal{A})$ are the maps in $T(X)$ that commute with the unique idempotent in $T(X)$ that has image $R$ and kernel $\rho$ (see [4] and [5]).

Problem. (Suggested by J.D. Mitchell.) Carry out (part of) the program outlined at the beginning of this section for the endomorphisms of various mathematical structures $\mathcal{A}$ with universe $X$, for example for the endomorphisms of a given partial order on $X$ or the endomorphisms of a given graph with $X$ as the set of vertices. (See [32].)

\section{Surjective transformations.}

It is easy to see that a transformation $\alpha \in T(X)$ is surjective if and only if it does not have any maximal right rays or finite branches.

Problem. Carry out (part of) the program outlined at the beginning of this section for the surjective transformations, for an infinite $X[X=\mathbb{N}]$. (See also [33].

\section{Acknowledgments}

The first author was partially supported by FCT through the following projects: PEst-OE/MAT/UI1043/2011, Strategic Project of Centro de Álgebra da Universidade de Lisboa; and PTDC/MAT/101993/2008, Project Computations in groups and semigroups . 


\section{References}

[1] J. M. André, J. Araújo, and J. Konieczny, Regular centralizers of idempotent transformations, Semigroup Forum 82 (2011), 307-318.

[2] J. Araújo, E. Dobson, and J. Konieczny, Automorphisms of endomorphism semigroups of reflexive digraphs, Math. Nachr. 283 (2010), 939-964.

[3] J. Araújo, M Kinyon, and J. Konieczny, Minimal paths in the commuting graphs of semigroups, European J. Combin. 32 (2011), 178-197.

[4] J. Araújo and J. Konieczny, Automorphism groups of centralizers of idempotents, J. Algebra 269 (2003), 227-239.

[5] J. Araújo and J. Konieczny, Semigroups of transformations preserving an equivalence relation and a cross-section, Comm. Algebra 32 (2004), 1917-1935.

[6] J. Araújo, and J. Konieczny, A method of finding automorphism groups of endomorphism monoids of relational systems, Discrete Math. 307 (2007), 1609-1620.

[7] J. Araújo and J. Konieczny, General theorems on automorphisms of semigroups and their applications, J. Aust. Math. Soc. 87 (2009), 1-17.

[8] J. Araújo and J. Konieczny, Directed graphs of inner translations of semigroups, to appear.

[9] J. Araújo and J. Mitchell, Relative ranks in the monoid of endomorphisms of an independence algebra. Monatsh. Math. 151 (2007), 1-10.

[10] C. Bates, D. Bundy, S. Perkins, and P. Rowley, Commuting involution graphs for symmetric groups, J. Algebra 266 (2003), 133-153.

[11] R. Diestel, Graph Theory, Third Edition, Springer-Verlag, Heidelberg, 2005.

[12] J. East, J. D. Mitchell, and Y. Péresse, Maximal subsemigroups of the semigroup of all functions on an infinite set, to appear.

[13] P. Hell and J. Nešetřil, Graphs and Homomorphisms, Oxford University Press, New York, 2004.

[14] P.M. Higgins, Digraphs and the semigroup of all functions on a finite set, Glasgow Math. J. 30 (1988), 41-57.

[15] J. M. Howie, The subsemigroup generated by the idempotents of a full transformation semigroup, J. London Math. Soc. 41 (1966), 707-716.

[16] J. M. Howie, Fundamentals of Semigroup Theory, Oxford Science Publications, Oxford, (1995).

[17] J.M. Howie, N. Ruškuc, and P.M. Higgins, On relative ranks of full transformation semigroups, Comm. Algebra 26 (1998), 733-748.

[18] A. Iranmanesh and A. Jafarzadeh, On the commuting graph associated with the symmetric and alternating groups, J. Algebra Appl. 7 (2008), 129-146. 
[19] D. Jakubíková, Systems of unary algebras with common endomorphisms. I, II, Czechoslovak Math. J. 29(104) (1979), 406-420, 421-429.

[20] V.A. Kolmykov, On the commutativity relation in a symmetric semigroup, Siberian Math. J. 45 (2004), 931-934.

[21] V.A., Kolmykov, Endomorphisms of functional graphs, Discrete Math. Appl. 16 (2006), 423427.

[22] V.A. Kolmykov, On commuting mappings, Math. Notes 86 (2009), 357-360.

[23] J. Konieczny, Green's relations and regularity in centralizers of permutations, Glasgow Math. J. 41 (1999), 45-57.

[24] J. Konieczny, Semigroups of transformations commuting with idempotents, Algebra Colloq. 9 (2002), 121-134.

[25] J. Konieczny, Semigroups of transformations commuting with injective nilpotents, Comm. Algebra 32 (2004), 1951-1969.

[26] J. Konieczny, Centralizers in the semigroup of injective transformations on an infinite set, Bull. Austral. Math. Soc. 82 (2010), 305-321.

[27] J. Konieczny, Infinite injective transformations whose centralizers have simple structure, Cent. Eur. J. Math. 9 (2011) 23-35.

[28] J. Konieczny and S. Lipscomb, Centralizers in the semigroup of partial transformations, Math. Japon. 48 (1998), 367-376.

[29] S.L. Lipscomb, The structure of the centralizer of a permutation, Semigroup Forum 37 (1988), 301-312.

[30] S. Lipscomb and J. Konieczny, Centralizers of permutations in the partial transformation semigroup, Pure Math. Appl. 6 (1995), 349-354.

[31] V.A. Liskovec and V.Z. Feĭnberg, On the permutability of mappings, Dokl. Akad. Nauk BSSR 7 (1963), 366-369. (Russian)

[32] J.D. Mitchell, M.P. Morayne, Y. Péresse, and M. Quick, Generating transformation semigroups using endomorphisms of preorders, graphs, and tolerances, Ann. Pure Appl. Logic 161 (2010), $1471-1485$.

[33] J. D. Mitchell and Y. Péresse, Generating countable sets of surjective functions, Fund. Math. 213 (2011), 67-93.

[34] M. Novotný, O jednom problému z teorie zobrazení, Publ. Fac. Sci. Univ. Massaryk 344 (1953), 53-64. (Czech)

[35] M. Novotný, Über Abbildungen von Mengen, Pacific J. Math. 13 (1963), 1359-1369. (German)

[36] L.A. Skornjakov, Unary algebras with regular endomorphism monoids, Acta Sci. Math. (Szeged) 40 (1978), 375-381. 
[37] M. Suzuki, Group Theory I, Springer-Verlag, New York, 1982.

[38] F. Szechtman, On the automorphism group of the centralizer of an idempotent in the full transformation monoid, Semigroup Forum 70 (2005),238-242.

[39] M.W. Weaver, On the commutativity of a correspondence and a permutation, Pacific J. Math. 10 (1960), 705-711.

[40] D. Zupnik, Cayley functions, Semigroup Forum 3 (1972), 349-358. 